\title{
Synthesis and antibacterial activity of Schiff bases and amines derived from alkyl 2-(2-formyl-4-nitrophenoxy)alkanoates
}

\author{
Agata Goszczyńska $^{1} \cdot$ Halina Kwiecieñ $^{1} \cdot$ Karol Fijałkowski $^{2}$
}

Received: 27 November 2014/ Accepted: 27 June 2015/Published online: 15 July 2015

(c) The Author(s) 2015. This article is published with open access at Springerlink.com

\begin{abstract}
A series of novel Schiff bases and secondary amines were obtained in good yields, as a result of the reductive amination of alkyl 2-(2-formyl-4-nitrophenoxy)alkanoates with both aniline and 4-methoxyaniline under established mild reaction conditions. Sodium triacetoxyborohydride as well as hydrogen in the presence of palladium on carbon were used as efficient reducing agents of the Schiff bases, in both direct and stepwise reductive amination processes. The Schiff bases, amines, and amine hydrochlorides were designed as potential antibacterial agents, and structure-activity relationship could be established following in vitro assays against Gram-positive and Gram-negative bacteria. The minimal inhibitory concentration and zone of inhibition were also determined. In these tests, some of Schiff bases and secondary amine hydrochlorides showed moderate-to-good activity against Gram-positive bacteria, including S. aureus, M. luteus, and S. mutans.
\end{abstract}

Electronic supplementary material The online version of this article (doi:10.1007/s00044-015-1397-6) contains supplementary material, which is available to authorized users.

Halina Kwiecień

halina.kwiecien@zut.edu.pl

Agata Goszczyńska

agata.goszczynska@zut.edu.pl

Karol Fijałkowski

karol.fijalkowski@zut.edu.pl

1 Department of Organic Synthesis and Drug Technology, West Pomeranian University of Technology, Szczecin, Al. Piastów 42, 71-065 Szczecin, Poland

2 Department of Immunology, Microbiology and Physiological Chemistry, West Pomeranian University of Technology, Szczecin, Al. Piastów 45, 70-311 Szczecin, Poland
Keywords Amines - Antibacterial activities - Esters · 2-(2-Formylphenoxy)alkanoic acids · Reduction . Reductive amination $\cdot$ Schiff bases

\section{Introduction}

The major problem in the effective antibacterial treatment is increasing resistance of microorganisms to currently available antimicrobial drugs. Therefore, the development of novel antimicrobial drugs is an active area of research. Most of compounds bearing an azomethine group exhibit antimicrobial (da Silva et al., 2011; Mohini et al., 2013; Shi et al., 2007), antioxidant, and antiproliferative properties (Cheng et al., 2010). Schiff bases, such as nitrofurantoin or nifuroxazide, are commonly applied in medicine as antibacterial agents (Sztanke et al., 2013). Additionally, a variety of phenoxyalkanoic acid derivatives are also known to possess a wide range of bioactivities (Hullar and Failla, 1969; Pattan et al., 2009; Kumar and Kumaresan, 2012), and some of the Schiff bases derived from 2-formylphenoxyacetic acids exhibit antibacterial properties (Bala et al., 2010; Iqbal et al., 2007). Further, aromatic secondary amines, as well as their salts, are also known to possess antimicrobial activity (Kitahara et al., 2004; Singh et al., 2011). Finally, secondary amines containing an aromatic nitro group exhibit an arginase inhibitory effect on vascular smooth muscle cell proliferation (Curtis et al., 2013).

A widely useful method for the synthesis of amines is reductive amination, which involves the reaction of aldehydes and ketones with ammonia or primary/secondary amines in the presence of a selective reducing agent (Tarasevich and Kozlov, 1999; Gomez et al., 2002). This process is considered direct when a carbonyl compound 
and an amine are mixed together with a reducing agent in a single operation. On the other hand, a stepwise reductive amination involves the pre-formation of the intermediate imine, followed by reduction in a separate step (AbdelMagid et al., 1996). A wide variety of reducing agents have been utilized for reductive amination; however, two methods have been used most commonly. The first method involves catalytic hydrogenation with platinum, palladium, ruthenium, cobalt or nickel catalysts (Klyuev and Khidekel, 1980; Petrisko and Krupka, 2005; Tripathi et al., 2008). The second method utilizes metal hydride reagents, mainly sodium borohydride (Panfilov et al., 2000), sodium triacetoxyborohydride (Abdel-Magid and Mehrman, 2006; Gribble, 2006), sodium or lithium cyanoborohydride (Borch et al., 1971; Grenga et al., 2009), and sodium borohydride modified with numerous polyvalent metal salts (Saxena et al., 2000; Saidi et al., 2007; Neidigh, et al., 1998) or activated by acids (Cho and Kang, 2005; Alinezhad et al., 2010).

Here, we report the synthesis of a series of Schiff bases and amines that were designed as potential antimicrobial agents. The synthesis involves the chemoselective reaction of primary amines with alkyl 2-(2-formyl-4-nitrophenoxy)alkanoates yielding Schiff bases bearing intact the ester group, as well as further reduction of the Schiff bases to the corresponding amino esters. Further, we have performed antibacterial screening of the obtained compounds against Gram-positive and Gram-negative bacteria and analyzed the influence of the electron-donating substituents such as methoxyl and amino groups in the phenyl rings, as well as length of hydrophobic side chain on the antibacterial activities.

\section{Results and discussion}

\section{Chemistry}

The desired alkyl 2-(2-formylphenoxy)alkanoates 1a-g were obtained in high yield by the condensation of adequately substituted 2-hydroxybenzaldehydes with alkyl 2-bromoalkanoates in the presence of potassium bicarbonate in dimethylformamide (Kwiecień, 2004). The Schiff bases $\mathbf{3 a}-\mathbf{l}$ were prepared by the reaction of alkyl 2-(2-formylphenoxy)alkanoates $\mathbf{1 a - g}$ with aniline (2a) and 4-methoxyaniline (2b) (Scheme 1). To determine the optimal conditions of the process, a series of reactions of methyl 2-(2-formyl-4-nitrophenoxy)butanoate (1a) with aniline (2a) were carried out using different solvents, such as tetrahydrofuran, 1,2-dichloroethane, and methanol or without any solvent. The influence of the ratio of reagents, reaction time, and catalyst on the yield was also assessed, as indicated in Table 1.
Based on these studies, we found that a nearly quantitative yield of the desired product was obtained when the reaction was carried out in methanol and in the presence of catalytic amount of the acetic acid, using equimolar ratio of the reactants (Entry 7, Table 1).

Under these established conditions, the reaction proceeded chemoselectively in the formyl group and leaving unchanged the ester group. Importantly, it was observed that the product 3a could be obtained in high yield by using an aprotic solvent, such as 1,2-dichloroethane in the presence of catalytic amounts of acetic acid. This is important for the direct reductive amination, which is more effective when it is carried out in 1,2-dichloroethane, as demonstrated later. The Schiff base was separated from the reaction mixture by dilution with water, followed by filtration of the precipitate.

Next, the Schiff bases $\mathbf{3 b}-\mathbf{l}$ were readily prepared by reactions of formyl esters $\mathbf{1 b}-\mathbf{g}$ with aniline (2a) and 4-methoxyaniline (2b), in the established reaction conditions (1:1 molar ratio of the formyl ester to amine, methanol as solvent, catalytic amount of acetic acid, room temperature, $3.5 \mathrm{~h}$ ). The crude products $(\mathbf{3 b}-\mathbf{l})$ were crystallized from methanol to yield stable crystals with high melting points (mp); yield: 72-95\%. Some of the Schiff bases exhibit a wide range of their melting points (see experimental section) that is probably caused by presence of $(E-)$ and (Z-) diastereoisomers in the solid state of the products.

The structures of novel Schiff bases $\mathbf{3 a}-\mathbf{l}$ were confirmed by gas chromatography mass spectrometry (GCMS), Fourier transform infrared spectroscopy (FTIR), and ${ }^{1} \mathrm{H}$ and ${ }^{13} \mathrm{C}$ nuclear magnetic resonance (NMR). The infrared spectra exhibited an intense absorption band in the range of 1623-1614 $\mathrm{cm}^{-1}$, characteristic of the azomethine groups. Additionally, intense bands, originating from the valence vibrations of the ester carbonyl group, were observed in the range $1756-1733 \mathrm{~cm}^{-1}(\mathrm{C}=\mathrm{O}$ stretch $)$ and 1208-1198 $\mathrm{cm}^{-1}$ (C-O stretch). Further, we observed a singlet of integration intensity equivalent to one hydrogen at $9.10-8.94 \mathrm{ppm}$ in the ${ }^{1} \mathrm{H}$ NMR spectra of the Schiff bases, indicating the presence of the azomethine proton $(-\mathrm{CH}=\mathrm{N}-)$.

Two methods were used for the reduction of Schiff bases. The first one was carried out using sodium triacetoxyborohydride (STAB) as a selective reducing agent of the imino group only and giving nitro amines $4 \mathbf{a}-$ f (Scheme 2). The second method consisted of catalytic reduction of both imino and nitro groups, resulting in the formation of diamine compounds $\mathbf{5 a}, \mathbf{c}-\mathbf{f}$ (Scheme 3 ).

To determine the optimal conditions for the reduction of Schiff bases with sodium triacetoxyborohydride to the nitro amines $\mathbf{4 a}-\mathbf{f}$, a series of reactions was carried out, starting from model compound $\mathbf{3 a}$ and using the following solvents: 
<smiles>[R]OC(=O)C([R])Oc1c([R])cc([N+](=O)[O-])cc1C=O</smiles>

$1 \mathrm{a}-\mathrm{g}$<smiles>[R]c1ccc(N)cc1</smiles>

2a: $\mathrm{R}=\mathrm{H}$

2b: $\mathrm{R}=\mathrm{OMe}$<smiles>[R]OC(=O)C([R])Oc1c([R])cc([N+](=O)[O-])cc1/N=C/c1ccc([R])cc1</smiles>

$3 a-1$

\begin{tabular}{cccccccccccc}
\hline $\mathbf{1 , 3}$ & $\mathrm{R}$ & $\mathrm{R}^{1}$ & $\mathrm{R}^{2}$ & $\mathrm{R}^{3}$ & $\begin{array}{c}\text { Yield of } \mathbf{3} \\
(\%)\end{array}$ & $\mathbf{3}$ & $\mathrm{R}$ & $\mathrm{R}^{1}$ & $\mathrm{R}^{2}$ & $\mathrm{R}^{3}$ & $\begin{array}{c}\text { Yield of } \mathbf{3} \\
(\%)\end{array}$ \\
\hline $\mathbf{a}$ & $\mathrm{H}$ & $\mathrm{H}$ & $\mathrm{Et}$ & $\mathrm{Me}$ & 95 & $\mathbf{g}$ & $\mathrm{H}$ & $\mathrm{OMe}$ & $\mathrm{H}_{2}$ & $\mathrm{Et}$ & 90 \\
$\mathbf{b}$ & $\mathrm{H}$ & $\mathrm{H}$ & $n-\mathrm{Pr}$ & $\mathrm{Me}$ & 76 & $\mathbf{h}$ & $\mathrm{OMe}$ & $\mathrm{H}$ & $\mathrm{Et}$ & $\mathrm{Me}$ & 83 \\
$\mathbf{c}$ & $\mathrm{H}$ & $\mathrm{H}$ & $n-\mathrm{Bu}$ & $\mathrm{Me}$ & 89 & $\mathbf{i}$ & $\mathrm{OMe}$ & $\mathrm{H}$ & $n-\mathrm{Bu}$ & $\mathrm{Me}$ & 72 \\
$\mathbf{d}$ & $\mathrm{H}$ & $\mathrm{OMe}$ & $\mathrm{Et}$ & $\mathrm{Me}$ & 85 & $\mathbf{j}$ & OMe & OMe & $\mathrm{Et}$ & $\mathrm{Me}$ & 89 \\
$\mathbf{e}$ & $\mathrm{H}$ & $\mathrm{OMe}$ & $n-\mathrm{Pr}$ & $\mathrm{Me}$ & 85 & $\mathbf{k}$ & $\mathrm{OMe}$ & $\mathrm{OMe}$ & $n-\mathrm{Pr}$ & $\mathrm{Me}$ & 90 \\
$\mathbf{f}$ & $\mathrm{H}$ & $\mathrm{OMe}$ & $n-\mathrm{Bu}$ & $\mathrm{Me}$ & 78 & $\mathbf{I}$ & OMe & OMe & $n$-Bu & $\mathrm{Me}$ & 76 \\
\hline
\end{tabular}

Scheme 1 Synthesis of Schiff bases from formyl esters $\mathbf{1 a}-\mathbf{g}$ and aniline (2a) or 4-methoxyaniline (2b)

Table 1 Synthesis of methyl 2-(4-nitro-2-((phenylimino)methyl)phenoxy)butanoate (3a)

\begin{tabular}{llllll}
\hline Entry & $\mathbf{1 a} / \mathbf{2 a}$ ratio $(\mathrm{mmol} / \mathrm{mmol})$ & Solvent $(\mathrm{mL})$ & & \multicolumn{2}{c}{ Reaction time $(\mathrm{h})^{\text {Yield }^{\mathrm{a}}(\%)}$} \\
\hline 1 & $1 / 1$ & THF & 12 & 24 & 91 \\
2 & $1 / 1$ & THF/MeOH & $6 / 6$ & 5.5 & 93 \\
3 & $1 / 1$ & DCE & 8 & 24 & 56 \\
4 & $1 / 1$ & $\mathrm{DCE}^{\mathrm{b}}$ & 8 & 24 & 91 \\
5 & $1 / 1.5$ & $\mathrm{DCE}^{\mathrm{b}}$ & 8 & 24 & 62 \\
6 & $1.5 / 1.5$ & $\mathrm{DCE} / \mathrm{MeOH}$ & $4 / 4$ & 72 & 99 \\
7 & $1 / 1$ & MeOH & 6 & 3.5 & $77^{\mathrm{b}}$ \\
8 & $1 / 1$ & - & - & 24 & \\
\hline
\end{tabular}

${ }^{a}$ Yield based on GC analysis of the reaction mixture

b Catalytic amount of acetic acid was added

${ }^{c}$ Reaction was carried out without any solvent

methanol, 1,2-dichloroethane, tetrahydrofuran, and $\mathrm{N}, \mathrm{N}$ dimethylformamide (Table 2). Utilizing methanol resulted in a low yield of amino ester, and neither extending the reaction time nor increasing the temperature improved the yield. Similarly, low yield was obtained using $N, N$ dimethylformamide in the presence of catalytic amounts of acetic acid. The best result was obtained using a 1:1.5 molar ratio of Schiff base/STAB in 1,2-dichloroethane and a catalytic amount of acetic acid. The reaction was carried out at room temperature for $4 \mathrm{~h}$. Next, the reaction mixture was neutralized with $5 \%$ aqueous solution of sodium bicarbonate, and the organic layer was separated and dried using magnesium sulfate. The product was isolated by solvent removal under reduced pressure and was recrystallized from methanol, to yield pure amino ester $\mathbf{4 a}$. Under these optimal conditions, the synthesis of compounds $\mathbf{4 b}$ f was readily achieved with moderate-to-good yields (Scheme 2). All of the synthesized amino esters $\mathbf{4 a - f}$ are novel compounds. Their structures were established by spectroscopic methods: GC-MS, FTIR, ${ }^{1} \mathrm{H}$, and ${ }^{13} \mathrm{C}$ NMR. The FTIR spectra exhibited an intense absorption band in the range of $3406-3385 \mathrm{~cm}^{-1}$, characteristic of the amine 
Scheme 2 Reduction of Schiff bases 3a-f with STAB into amines $\mathbf{4 a - f}$<smiles>[R]c1cc([N+](=O)[O-])cc(/C=N/c2ccccc2)c1OC([R])C(=O)OC</smiles>

3a-f

4a-f

\begin{tabular}{cccccccc}
\hline 3, 4 & $\mathrm{R}^{1}$ & $\mathrm{R}^{2}$ & Yield of 4 (\%) & $\mathbf{3 , 4}$ & $\mathrm{R}^{1}$ & $\mathrm{R}^{2}$ & Yield of 4 (\%) \\
\hline a & $\mathrm{H}$ & $\mathrm{Et}$ & 60 & $\mathbf{d}$ & $\mathrm{OMe}$ & $\mathrm{Et}$ & 55 \\
$\mathbf{b}$ & $\mathrm{H}$ & $n-\mathrm{Pr}$ & 60 & $\mathbf{e}$ & OMe & $n-\mathrm{Pr}$ & 55 \\
$\mathbf{c}$ & $\mathrm{H}$ & $n-\mathrm{Bu}$ & 55 & $\mathbf{f}$ & OMe & $\mathrm{n}-\mathrm{Bu}$ & 85 \\
\hline
\end{tabular}<smiles>[R]c1cc([N+](=O)[O-])cc(/C=N/c2ccccc2)c1OC([R])C(=O)OC</smiles>

3a, 3c-f

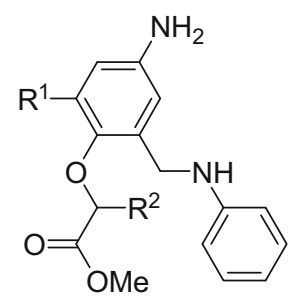

$5 a, 5 c-f$

\begin{tabular}{cccccc}
\hline $\mathbf{3 , 5}$ & $\mathrm{R}^{1}$ & $\mathrm{R}^{2}$ & $\mathbf{3 , 5}$ & $\mathrm{R}^{1}$ & $\mathrm{R}^{2}$ \\
\hline $\mathbf{a}$ & $\mathrm{H}$ & $\mathrm{Et}$ & $\mathbf{e}$ & $\mathrm{OMe}$ & $n-\mathrm{Pr}$ \\
$\mathbf{c}$ & $\mathrm{H}$ & $n-\mathrm{Bu}$ & $\mathbf{f}$ & $\mathrm{OMe}$ & $n-\mathrm{Bu}$ \\
$\mathbf{d}$ & $\mathrm{OMe}$ & $\mathrm{Et}$ & & & \\
\hline
\end{tabular}

Scheme 3 Catalytic reduction of Schiff bases $\mathbf{5 a}$ and $\mathbf{5 c - f}$

groups. Additionally, intense bands, originating from the valence vibrations of the ester carbonyl group, were observed in the range $1751-1742 \mathrm{~cm}^{-1}(\mathrm{C}=\mathrm{O}$ stretch $)$ and $1209-1200 \mathrm{~cm}^{-1}$ (C-O stretch). In the ${ }^{1} \mathrm{H}$ NMR spectra of the amino esters, the multiplet at $4.70-4.31 \mathrm{ppm}$ is consist of two doublets and broad signal which are derived from two protons of $\mathrm{CH}_{2}$ and a one proton of $\mathrm{NH}$ group, respectively. The deuterium exchange experiment with $\mathrm{D}_{2} \mathrm{O}$ was performed to confirm the presence of amine proton (see Supporting Information).

Reduction of both nitro and azomethine groups of $\mathbf{3 a}$ and 3c-f was carried out in mild conditions: in methanol with the addition of dimethoxyethane (DME), using a 1:0.1 weight ratio of Schiff base to catalyst, $10 \% \mathrm{Pd} / \mathrm{C}$ (Scheme 3). The reaction was completed after $7 \mathrm{~h}$, and the

Table 2 Reduction of Schiff base $\mathbf{3 a}$ to amine $\mathbf{4 a}$ with STAB

\begin{tabular}{|c|c|c|c|c|c|c|}
\hline \multirow{2}{*}{$\frac{\text { Entry }}{1}$} & \multirow{2}{*}{$\begin{array}{l}\text { 4a/STAB ratio }(\mathrm{mmol} / \mathrm{mmol}) \\
1 / 1.4\end{array}$} & \multicolumn{2}{|c|}{ Solvent (mL) } & \multirow{2}{*}{$\frac{\text { Reaction temp. }}{100{ }^{\circ} \mathrm{C}}$} & \multirow{2}{*}{$\begin{array}{l}\text { Reaction time (h) } \\
5\end{array}$} & \multirow{2}{*}{$\frac{\text { Yield }^{\mathrm{a}}(\%)}{21}$} \\
\hline & & $\mathrm{DMF}^{\mathrm{c}}$ & 14 & & & \\
\hline 2 & $1 / 1.4$ & $\mathrm{MeOH}$ & 44 & $\mathrm{rt}$ & 3 & 23 \\
\hline 3 & $1 / 1.4$ & $\mathrm{MeOH}$ & 44 & reflux & 4 & 27 \\
\hline 4 & $1 / 1.4$ & THF & 20 & reflux & 4 & 65 \\
\hline 5 & $1 / 1.7$ & DCE & 20 & $\mathrm{rt}$ & 6 & 86 \\
\hline 6 & $1 / 1.5$ & $\mathrm{DCE}^{\mathrm{b}}$ & 17 & $\mathrm{rt}$ & 4 & 93 \\
\hline 7 & $1 / 2.0$ & $\mathrm{DCE}^{\mathrm{b}}$ & 17 & $\mathrm{rt}$ & 4 & 81 \\
\hline 8 & $1 / 1.5$ & $\mathrm{DCE}^{\mathrm{c}}$ & 17 & $\mathrm{rt}$ & 4 & 84 \\
\hline
\end{tabular}

${ }^{a}$ Yield based on GC analysis of reaction mixture

b Added $\mathrm{AcOH}(0.1 \mathrm{~mL})$

c Added $\mathrm{AcOH}(0.2 \mathrm{~mL})$ 
Table 3 Catalytic reduction of Schiff bases $\mathbf{3 a}$ and $\mathbf{3 c}-\mathbf{f}$ with hydrogen

\begin{tabular}{|c|c|c|c|c|c|}
\hline 3 & $3 /(10 \% \mathrm{Pd} / \mathrm{C})$ ratio $(\mathrm{g} / \mathrm{g})$ & $\mathrm{MeOH}(\mathrm{mL})$ & $\mathrm{DME}(\mathrm{mL})$ & 5 & Yield $(\%)$ \\
\hline $\mathbf{a}$ & $0.31 / 0.031$ & 40 & 10 & $\mathbf{a}$ & 85 \\
\hline c & $0.20 / 0.020$ & 35 & 7 & c & 82 \\
\hline d & $0.20 / 0.020$ & 35 & 20 & d & 71 \\
\hline $\mathbf{e}$ & $0.36 / 0.036$ & 40 & 10 & e & 84 \\
\hline f & $0.16 / 0.016$ & 35 & 7 & f & 74 \\
\hline
\end{tabular}

Table 4 Synthesis of $\mathbf{4 a}$ via the direct reductive amination of methyl 2-(2-formyl-4-nitrophenoxy)butanoate (1a)

\begin{tabular}{lllll}
\hline $\mathbf{1 a} / \mathbf{2 a} / \mathrm{STAB}$ ratio $(\mathrm{mmol} / \mathrm{mmol} / \mathrm{mmol})$ & Solvent $(\mathrm{mL})$ & Catalyst & Reaction time $(\mathrm{h})^{\text {Yield }^{\text {a }}(\%)}$ \\
\hline $1 / 1 / 1$ & THF & - & 50 & 42 \\
$1 / 1 / 1.45$ & THF & - & 50 & 61 \\
$1 / 1 / 1$ & DCE & - & 50 & 91 \\
$1 / 1.5 / 1.5$ & DCE & - & 50 & 98 \\
$1 / 1 / 1.5$ & DCE & - & 4 & 59 \\
$1 / 1 / 1.5$ & DCE & - & 24 & 86 \\
$1 / 1 / 1.5$ & DCE & Amberlyst-15 & 4 & 89 \\
$1 / 1 / 1.5$ & DCE & AcOH & 4 & 98 \\
\hline
\end{tabular}

a Yield based on GC analysis

products $\mathbf{5 a}$ and $\mathbf{5} \mathbf{c}-\mathbf{f}$ were obtained after removing of the solvent under vacuum. Diamines $\mathbf{5 a}, \mathbf{c}-\mathbf{f}$ were obtained as brown semi-solids, in good yield (71-86\%, Table 3).

The FTIR spectra of $\mathbf{5 a}, \mathbf{c}-\mathbf{f}$ exhibit an intensive absorption band in the range of $3410-3374 \mathrm{~cm}^{-1}$, characteristic of the amine groups. Additionally, intensive bands, originating from the valence vibrations of the ester carbonyl group, were observed in the range $1735-1737 \mathrm{~cm}^{-1}$. Finally, the signal at 9.10-8.94 ppm in the ${ }^{1} \mathrm{H}$ NMR spectra, associated with azomethine group, disappeared and instead signals at ranges $3.90-3.53$ and $1.51-0.78 \mathrm{ppm}$ were observed, indicating the presence of the amine protons.

Subsequently, reductive amination of methyl 2-(2formylphenoxy)alkanoate 1a-f was investigated as a onestep process. Synthesis of amines via direct reductive amination is very useful, because it does not require isolating the intermediate Schiff bases. This greatly speeds up the process of synthesis and limits losses associated with isolation of the intermediates.

To determine optimal conditions for the direct reductive amination, the model formyl ester 1a was reacted with aniline (2a) in the presence of sodium triacetoxyborohydride as the reducing agent. The reactions were conducted at ambient temperature, using different solvents, and changing the molar ratio of the reactants and reaction times (Table 4).
The highest yield of the desired product was achieved when the reaction was performed in 1,2-dichloroethane with a catalytic amount of acetic acid for $4 \mathrm{~h}$, with an equimolar ratio of formyl ester and aniline and $1.5 \mathrm{~mol}$ excess of the catalyst.

The direct reductive amination of $\mathbf{1 b}-\mathbf{f}$ was carried out using the same conditions as for 1a, resulting in good yields (71-85\%) of amines $4 \mathbf{b}-\mathbf{f}$ (Scheme 4). Finally, amino esters $\mathbf{4 a - f}$ were converted into their hydrochloride salts.

\section{Microbiology}

All of the synthesized compounds were screened for antibacterial activity against selected clinically important Gram-positive (S. aureus, M. luteus, S. mutans, E. faecalis) and Gram-negative (E. coli, P. aeruginosa, A. baumannii) bacteria by the disk diffusion method. Acetone was used as solvent for Schiff bases, and DMSO as solvent for amines and hydrochloride salts of amines. The antibiotic ciprofloxacin $(5 \mathrm{mg} / \mathrm{mL})$ was used as a positive control. The results of antibacterial screening indicate that four of eleven tested Schiff bases 3a, 3c-e exhibit varied activity against Gram-positive bacteria, including $S$. aureus, and $S$. mutans. The bacterial inhibition zone values of the Schiff bases are summarized in Table 5 . 
Scheme 4 Direct reductive amination of formyl esters 1af to $4 \mathbf{a}-\mathbf{f}$<smiles>[R]C(=O)C([R])Oc1c([R])cc([N+](=O)[O-])cc1C=O</smiles>

\begin{tabular}{cccccccc}
\hline $\mathbf{1 , 4}$ & $\mathrm{R}^{1}$ & $\mathrm{R}^{2}$ & Yield of $\mathbf{4}(\%)$ & $\mathbf{1 , 4}$ & $\mathrm{R}^{1}$ & $\mathrm{R}^{2}$ & Yield of $\mathbf{4}(\%)$ \\
\hline $\mathbf{a}$ & $\mathrm{H}$ & $\mathrm{Et}$ & 85 & $\mathbf{d}$ & OMe & $\mathrm{Et}$ & 84 \\
$\mathbf{b}$ & $\mathrm{H}$ & $n-\mathrm{Pr}$ & 82 & $\mathbf{e}$ & OMe & $n-\mathrm{Pr}$ & 78 \\
$\mathbf{c}$ & $\mathrm{H}$ & $n-\mathrm{Bu}$ & 71 & $\mathbf{f}$ & OMe & $n-\mathrm{Bu}$ & 74 \\
\hline
\end{tabular}

Table 5 Antibacterial activity ${ }^{a}$ of the Schiff bases $\mathbf{3 a}$ and $\mathbf{3 c}-\mathbf{e}$ at $10 \mathrm{mg} / \mathrm{mL}$ concentration

\begin{tabular}{llllc}
\hline Compound & S. aureus & & & S. mutans \\
\cline { 2 - 5 } & MSSA ATCC 25923 & MRSA ATCC 43300 & MLSB clinical isolate & 10 \\
3a & 13 & 8 & 9 & 7 \\
3c & 7 & 7 & 8 & - \\
3d & 10 & 15 & 12 & 7 \\
3e & 8 & 13 & 13 & 28 \\
ciprofloxacin & 25 & 26 & 27 & 2 \\
\hline
\end{tabular}

a Zone of inhibition in mm; '-' means not sensitive (no antimicrobial activity was recorded)

Table 6 Minimum inhibitory concentration (MIC, $\mathrm{mg} / \mathrm{mL}$ ) of Schiff bases $\mathbf{3 a}, \mathbf{c}-\mathbf{e}$

\begin{tabular}{lllll}
\hline Compound & \multicolumn{2}{l}{ S. aureus } & & \multicolumn{2}{c}{$\begin{array}{c}\text { mutans } \\
\text { clinical isolate }\end{array}$} \\
\cline { 2 - 5 } & MSSA ATCC 25923 & MRSA ATCC 43300 & MLSB clinical isolate & 0.50 \\
3a & 0.10 & 0.10 & 0.10 & 0.50 \\
3c & 0.50 & 0.25 & 0.25 & 1.00 \\
3d & 0.25 & 0.25 & 0.25 & 1.00 \\
3e & 0.25 & 0.10 & 0.10 & \\
\hline
\end{tabular}

Schiff base 3d exhibited antibacterial activity only against $S$. aureus strains and caused the strongest inhibition of the growth of methicillin-resistant $S$. aureus. Schiff base 3a, on the other hand, caused the strongest inhibition of the growth of $S$. mutans. It should also be mentioned that the synthesized Schiff bases did not inhibit the growth of E. faecalis and M. luteus. Further, introducing the methoxyl group into Schiff base structure has a significant impact on antibacterial activity. No antibacterial activity was observed for the Schiff bases containing methoxyl in $N$-substituted phenyl ring $(\mathbf{3 h}-\mathbf{l}, \mathbf{R}=\mathrm{OMe})$. Likewise, the presence of methoxyl group at 2-position in 3d structure abolishes activity against $S$. mutans, in contrast to $S$. aureus, where activity increases against MRSA. Increasing alkyl, hydrophobic chain length results in decreased antibacterial activity, except for the influence of $\mathbf{3 e}$ on S. mutans.

Followed the screening, the minimum inhibitory concentration (MIC) was determined for the Schiff bases that demonstrated activity against specific species of bacteria. The results are presented in Table 6.

Next, it was observed that amino esters 4a-f did not show any antimicrobial activity, while their hydrochlorides showed good inhibition of the Gram-positive bacteria (Table 7). The most active were those without the methoxyl groups, but no influence of hydrophobic side chain on the 
Table 7 Antibacterial activity ${ }^{\mathrm{a}}$ of amino esters $\mathbf{4 a}-\mathbf{f}$ at $10 \mathrm{mg} / \mathrm{mL}$ concentration

\begin{tabular}{|c|c|c|c|c|c|c|}
\hline \multirow[t]{2}{*}{ Compound } & \multirow{2}{*}{$\begin{array}{l}\text { E. faecalis } \\
\text { ATCC } 29212\end{array}$} & \multicolumn{3}{|l|}{ S. aureus } & \multirow{2}{*}{$\begin{array}{l}\text { M. luteus } \\
\text { PCM } 1944\end{array}$} & \multirow{2}{*}{$\begin{array}{l}\text { S. mutans } \\
\text { clinical isolate }\end{array}$} \\
\hline & & MSSA ATCC 25923 & MRSA ATCC 43300 & MLSB clinical isolate & & \\
\hline $\mathbf{4 a} \cdot \mathrm{HCl}$ & 12 & 8 & - & 8 & 15 & 11 \\
\hline $4 \mathbf{b} \cdot \mathrm{HCl}$ & 12 & 12 & 7 & 7 & 14 & 14 \\
\hline $4 \mathbf{c} \cdot \mathrm{HCl}$ & 17 & 16 & 13 & 8 & 17 & 21 \\
\hline $\mathbf{4 e} \cdot \mathrm{HCl}$ & 8 & - & - & - & 8 & - \\
\hline $\mathbf{4 f} \cdot \mathrm{HCl}$ & 13 & 13 & 14 & 12 & 15 & 15 \\
\hline ciprofloxacin & 20 & 25 & 26 & 27 & 22 & 28 \\
\hline
\end{tabular}

a Zone of inhibition in mm; '-' no antimicrobial activity was recorded

Table 8 Minimum inhibitory concentration (MIC, $\mathrm{mg} / \mathrm{mL}$ ) of hydrochloride salts of the amino esters $4 \mathbf{a}-\mathbf{f}$

\begin{tabular}{|c|c|c|c|c|c|c|}
\hline & \multicolumn{6}{|l|}{ Gram-positive } \\
\hline & \multirow{2}{*}{$\begin{array}{l}\text { E. faecalis } \\
\text { ATCC } 29212\end{array}$} & \multicolumn{3}{|l|}{ S. aureus } & \multirow{2}{*}{$\begin{array}{l}\text { M. luteus } \\
\text { PCM } 1944\end{array}$} & \multirow{2}{*}{$\begin{array}{l}\text { S. mutans } \\
\text { clinical isolate }\end{array}$} \\
\hline & & MSSA ATCC 25923 & MRSA ATCC 43300 & MLSB clinical isolate & & \\
\hline $\mathbf{4 a} \cdot \mathrm{HCl}$ & 0.50 & 0.50 & NT & 1.00 & 0.50 & 0.50 \\
\hline $4 \mathbf{b} \cdot \mathrm{HCl}$ & 0.25 & 0.10 & 0.50 & 1.00 & 0.05 & 0.25 \\
\hline $4 \mathbf{c} \cdot \mathrm{HCl}$ & 0.05 & 0.10 & 0.25 & 0.50 & 0.05 & 0.10 \\
\hline $4 \mathbf{e} \cdot \mathrm{HCl}$ & 0.50 & NT & NT & NT & 1.00 & NT \\
\hline $\mathbf{4 f} \cdot \mathrm{HCl}$ & 0.05 & 0.01 & 0.25 & 0.25 & 0.05 & 0.10 \\
\hline
\end{tabular}

NT means not tested

antibacterial activity was observed. The lack of inhibition for amino esters might be caused by the presence of an intramolecular hydrogen bond that can be formed between amino proton and carbonyl group in the amino esters.

The broadest spectrum of antibacterial activity was noted for $\mathbf{4} \mathbf{c} \cdot \mathrm{HCl}$. This compound inhibited the growth of all Gram-positive bacteria and was the only compound synthesized in these studies that inhibited the growth of the Gram-negative A. baumannii. The MIC of hydrochloride salts of $\mathbf{4 a}-\mathbf{f}$ is given in Table 8 .

Three other hydrochloride salts of amino esters, $\mathbf{4 a} \cdot \mathrm{HCl}$, $\mathbf{4 b} \cdot \mathrm{HCl}, \mathbf{4 f} \cdot \mathrm{HCl}$, inhibited the growth of all Gram-positive bacteria, having MICs of $0.50-1.00,0.05-1.0$, and $0.01-0.25 \mathrm{mg} / \mathrm{mL}$, respectively, for each bacterial strain. Moderate antibacterial activity was also recorded for $4 \mathbf{e} \cdot \mathrm{HCl}$, which inhibited the growth of E. faecalis and M. luteus. The most encouraging results against Gram-positive bacteria were obtained for compounds $\mathbf{4 c} \cdot \mathrm{HCl}$ and $\mathbf{4 f} \cdot \mathrm{HCl}$ having MICs of $0.05-0.50$ and $0.01-0.25 \mathrm{mg} / \mathrm{mL}$, respectively.

The structure-activity relationships of the tested compounds can be summarized as follows: (1) in series of the Schiff bases, the presence of methoxyl group at the $\mathrm{N}$ phenyl ring has a significant negative impact on their antibacterial activity; (2) in the same series, elongation of hydrophobic, alkyl chain causes the decrease in antibacterial activity against $S$. aureus MSSA and MRSA; (3) reduction of azomethine group to amine causes loss of activity against all of the tested microorganism; (4) in series of amine hydrochlorides, the presence of methoxyl group at the $N$-phenyl ring has the same negative impact on their antibacterial activity as in the case of Schiff bases; opposite effect is observed for elongation of hydrophobic, alkyl chain that causes the increase in antibacterial activity.

Lack of activity of the tested substances against Gramnegative bacteria could be explained by the differences in the structure of the cell walls of Gram-positive and Gramnegative microorganisms. In most Gram-positive bacteria, the cell wall consists of many layers of peptidoglycan, forming a thick, rigid structure. The cell walls of Gramnegative bacteria consist of one or a very few layers of peptidoglycan and a lipid-rich outer membrane (Beveridge, 1999). However, the mechanism responsible for the antibacterial activity of examined compounds is not known at the moment; work is in progress to clarify in detail the mechanism of antibacterial action, as well as the design of more effective compounds.

\section{Conclusion}

We demonstrate simple and efficient methods for both stepwise and direct reductive amination of 2-(2-formyl-4nitrophenoxy)alkanoic acid derivatives, yielding secondary $\mathrm{N}$-arylated amines via Schiff bases under mild conditions. 
Fig. 1 Numbering for ${ }^{13} \mathrm{C}$ NMR spectra

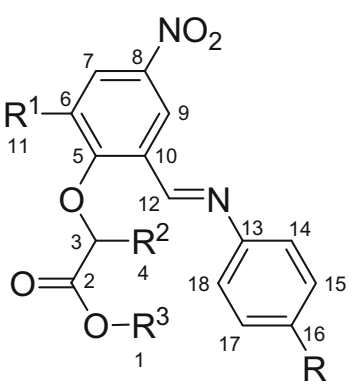

3a-I

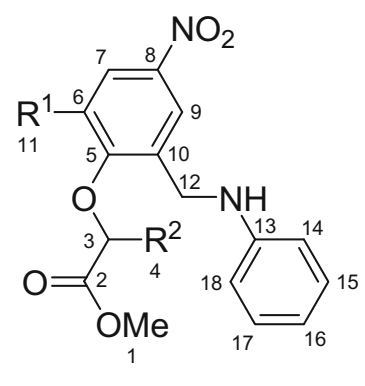

4a-f

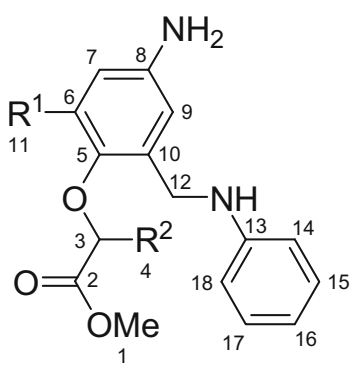

$5 a, c-f$
The reduction step was performed utilizing sodium triacetoxyborohydride, as well as catalytic hydrogenation using palladium $(0)$ catalyst. Our antibacterial screening assay indicates that some of Schiff bases and secondary amine hydrochlorides possess moderate-to-good activity against Gram-positive bacteria, including S. aureus, $M$. luteus, and S. mutans. In a series of Schiff bases, we observe some of the influence of chain length and presence of methoxyl group on the antibacterial activity. Further modification of the selected compounds based on the information obtained from these results, as well as molecular modeling and structure-activity relationship studies, are in progress.

\section{Materials and methods}

\section{Chemistry}

${ }^{1} \mathrm{H}$ and ${ }^{13} \mathrm{C}$ NMR spectra were recorded on a TM Bruker DPX $400(400 \mathrm{MHz})$ instrument, with $\mathrm{CDCl}_{3}$ solvent. Chemical shifts $\delta$ are given from TMS $(0 \mathrm{ppm})$, as an internal standard for ${ }^{1} \mathrm{H} \mathrm{NMR}$, and $\mathrm{CDCl}_{3}$ (77.0 ppm), for ${ }^{13} \mathrm{C}$ NMR (100 MHz). Mass spectra were obtained using an Agilent Technologies $6890 \mathrm{~N}$ apparatus, equipped with a mass detector 5973 Network and $30 \mathrm{~m} \times 0.25 \mathrm{~mm}$ capillary column, filled with a $0.25-\mu \mathrm{m}$ film of $5 \% \mathrm{MePh}$ silicate. Fourier transform infrared-attenuated total reflection spectroscopy (FTIR-ATR) was performed using a Nexus spectrometer with Golden Gate (ATR) (Thermo Nicolet Corp.). Samples were dried at $60{ }^{\circ} \mathrm{C}$, under vacuum for $24 \mathrm{~h}$, and 32 scans were averaged across the spectral range of $400-4000 \mathrm{~cm}^{-1}$. All melting points were determined using a Boetius apparatus and are uncorrected.

Most of the reagents and solvents were purchased in commercially available grade purity. 2-Hydroxy-3-methoxy-5-nitrobenzaldehyde (mp 140-142 ${ }^{\circ} \mathrm{C}$ ) and 2-hydroxy5-nitrobenzaldehyde (mp $\left.129-131^{\circ} \mathrm{C}\right)$ were obtained by nitration of appropriate 2-hydroxy-3-methoxybenzaldehyde or 2-hydroxybenzaldehyde with $100 \%$ nitric acid in acetic acid solution (Kwiecień and Szychowska, 2007), although they are commercially available. Methyl 2-(2formyl-4-nitrophenoxy)alkanoates $\mathbf{1 a - c}$ were prepared starting from appropriate 2-bromoesters and 2-hydroxy-5nitrobenzaldehyde according to the literature (Kwiecien, 2004). Methyl 2-(2-formyl-6-methoxy-4-nitrophenoxy) alkanoates 1d-f and ethyl 2-formyl-6-methoxy-4-nitrophenoxy)acetate $1 \mathrm{~g}$ were obtained starting from 2-hydroxy3-methoxy-5-nitrobenzaldehyde and appropriate 2-bromoesters according to procedures in Kwiecień (2004) and Kwiecień and Szychowska (2007), respectively (Fig. 1).

General procedure for the synthesis of methyl 2-(4nitro-2-((phenylimino)methyl)phenoxy)alkanoate $(3 a-1)$

A mixture of alkyl 2-(2-formyl-4-nitrophenoxy)alkanoate $(\mathbf{1 a}-\mathbf{g})(1.87 \mathrm{mmol})$, aniline (2a) or $p$-methoxyaniline $(\mathbf{2 b})$ $(1.87 \mathrm{mmol})$, methanol $(50 \mathrm{~mL})$, and acetic acid $(0.2 \mathrm{~mL})$ was stirred magnetically at room temperature for $3.5 \mathrm{~h}$. Then, the reaction mixture was poured into water $(27 \mathrm{~mL})$ and allowed to stand at $5-7^{\circ} \mathrm{C}$ (in a refrigerator) for $24 \mathrm{~h}$. The precipitate was filtered off, washed with water, and recrystallized from hot methanol to give $\mathbf{3 a}-\mathbf{l}$.

Methyl 2-(4-nitro-2-((phenylimino)methyl)phenoxy)butanoate (3a) Colorless solid (MeOH) This compound (3a) was prepared from methyl 2-(2-formyl-4-nitrophenoxy)butanoate (1a) $(1.87 \mathrm{mmol}, 0.50 \mathrm{~g})$ and aniline (2a) $(1.87 \mathrm{mmol}, 0.17 \mathrm{~g})$ according to the general procedure. The product obtained as a colorless solid was purified from methanol. $0.61 \mathrm{~g}(95 \%)$; mp $111-117^{\circ} \mathrm{C}$; FTIR (neat) $v_{\max }: 3081,2975,1744,1620,1585,1510,1340,1210$, $1077 \mathrm{~cm}^{-1} ;{ }^{1} \mathrm{H}$ NMR $\left(\mathrm{CDCl}_{3}, 400 \mathrm{MHz}\right): \delta=9.09(\mathrm{~d}$, $J=2.9 \mathrm{~Hz}, 1 \mathrm{H}, \mathrm{Ar}), 8.95(\mathrm{~s}, 1 \mathrm{H}, \mathrm{N}=\mathrm{CH}), 8.27(\mathrm{dd}$, $J=2.9,9.1 \mathrm{~Hz}, 1 \mathrm{H}, \mathrm{Ar}), 7.49-7.38$ (m, 2H, Ar), 7.30-7.24 $(\mathrm{m}, 3 \mathrm{H}, \mathrm{Ar}), 6.86(\mathrm{~d}, J=9.1 \mathrm{~Hz}, 1 \mathrm{H}, \mathrm{Ar}), 4.83(\mathrm{t}$, $J=6.0 \mathrm{~Hz}, 1 \mathrm{H}, \mathrm{CH}), 3.78\left(\mathrm{~s}, 3 \mathrm{H}, \mathrm{OCH}_{3}\right), 2.18-2.05(\mathrm{~m}$, $\left.2 \mathrm{H}, \mathrm{CH}_{2}\right), 1.12\left(\mathrm{t}, J=7.4 \mathrm{~Hz}, 3 \mathrm{H}, \mathrm{CH}_{3}\right) ;{ }^{13} \mathrm{C} \mathrm{NMR}$ 
$\left(\mathrm{CDCl}_{3}, 101 \mathrm{MHz}\right): \delta=170.5$ (C, C-2), $161.7(\mathrm{CH}, \mathrm{C}-12)$, 153.4 (C, C-5), 151.6 (C, C-13), 142.4 (C, C-8), 129.3 (C, C-15, C-17), 127.4 (C, C-7), 126.6 (C, C-9), 126.2 (C, C-10), 124.0 (C, C-16), 121.1 (C, C-14, C-18), 112.5 (C, C-6), $78.4(\mathrm{CH}, \mathrm{C}-3), 52.6\left(\mathrm{OCH}_{3}, \mathrm{C}-1\right), 26.0\left(\mathrm{CH}_{2} \mathrm{CH}_{3}\right.$, C-4), $9.7\left(\mathrm{CH}_{2} \mathrm{CH}_{3}, \mathrm{C}-4\right)$; GCMS m/z $342[\mathrm{M}]^{+}(32), 327$ (9), 283 (32), 250 (18), 225 (18), 222 (5), 195 (23), 190 (8), 179 (7), 175 (100), 145 (23), 139 (7), 104 (17), 93 (22), 77 (48), 59 (29), 51 (8); Anal. Calcd for $\mathrm{C}_{18} \mathrm{H}_{18} \mathrm{~N}_{2} \mathrm{O}_{5}$ : C, 63.15; H, 5.30; N, 8.18. Found: 63.12; H, 5.40; N, 8.15.

Methyl 2-(4-nitro-2-((phenylimino)methyl)phenoxy)pentanoate (3b) Colorless solid $(\mathrm{MeOH})$ This compound (3b) was prepared from methyl 2-(2-formyl-4-nitrophenoxy)pentanoate (1b) $(1.78 \mathrm{mmol}, 0.50 \mathrm{~g})$ and aniline (2a) $(1.78 \mathrm{mmol}, 0.162 \mathrm{~g})$ according to the general procedure. The product obtained as a colorless solid was purified from methanol. $0.48 \mathrm{~g}(76 \%)$; mp $84-87^{\circ} \mathrm{C}$; FTIR (neat) $v_{\max }$ : 3102 , 2962, 1733, 1614, 1579, 1515, 1341, 1268, $1078 \mathrm{~cm}^{-1}$; ${ }^{1} \mathrm{H}$ NMR $\left(\mathrm{CDCl}_{3}, 400 \mathrm{MHz}\right): \delta=9.10(\mathrm{~d}$, $J=2.9 \mathrm{~Hz}, 1 \mathrm{H}$, Ar $), 8.94(\mathrm{~s}, 1 \mathrm{H}, \mathrm{N}=\mathrm{CH}), 8.28(\mathrm{dd}$, $J=2.9,9.1 \mathrm{~Hz}, 1 \mathrm{H}, \mathrm{Ar}), 7.47-7.41$ (m, 2H, Ar), 7.32-7.25 $(\mathrm{m}, 3 \mathrm{H}, \mathrm{Ar}), 6.87(\mathrm{~d}, J=9.1 \mathrm{~Hz}, 1 \mathrm{H}, \mathrm{Ar}), 4.88(\mathrm{dd}$, $J=4.9,7.6 \mathrm{~Hz}, 1 \mathrm{H}, \mathrm{CH}), 3.79\left(\mathrm{~s}, 3 \mathrm{H}, \mathrm{OCH}_{3}\right), 2.14-1.98$ $\left(\mathrm{m}, 2 \mathrm{H}, \mathrm{CH}_{2}\right), \quad 1.65-1.50\left(\mathrm{~m}, 2 \mathrm{H}, \mathrm{CH}_{2}\right), 1.01(\mathrm{t}$, $\left.J=7.4 \mathrm{~Hz}, 3 \mathrm{H}, \mathrm{CH}_{3}\right) ;{ }^{13} \mathrm{C} \mathrm{NMR}\left(\mathrm{CDCl}_{3}, 101 \mathrm{MHz}\right)$ : $\delta=170.7(\mathrm{C}, \mathrm{C}-2), 161.7(\mathrm{CH}, \mathrm{C}-12), 153.5(\mathrm{C}, \mathrm{C}-5)$, 151.6 (C, C-13), 142.3 (C, C-8), 129.2 (C, C-15, C-17), 127.4 (C, C-7), 126.6 (C, C-9), 126.1 (C, C-10), 124.0 (C, C-16), 121.1 (C, C-14, C-18), 112.5 (C, C-6), 77.2 (CH, $\mathrm{C}-3), 52.6\left(\mathrm{OCH}_{3}, \mathrm{C}-1\right), 34.5\left(\mathrm{CH}_{2} \mathrm{CH}_{2} \mathrm{CH}_{3}, \mathrm{C}-4\right), 18.6$ $\left(\mathrm{CH}_{2} \mathrm{CH}_{2} \mathrm{CH}_{3}, \mathrm{C}-4\right), 13.7\left(\mathrm{CH}_{2} \mathrm{CH}_{2} \mathrm{CH}_{3}, \mathrm{C}-4\right) ; \mathrm{GCMS} \mathrm{m} / \mathrm{z}$ $356[\mathrm{M}]^{+}$(55), 327 (55), 297 (32), 264 (23), 241 (16), 225 (20), 204 (9), 195 (29), 189 (100), 179 (7), 167 (19), 145 (12), 130 (5), 118 (5), 104 (16), 93 (29), 77 (42), 59 (19); Anal. Calcd for $\mathrm{C}_{19} \mathrm{H}_{20} \mathrm{~N}_{2} \mathrm{O}_{5}$ : C, 64.04; H, 5.66; N, 7.86. Found: C, 64.14; H, 5.68; N, 7.82.

Methyl 2-(4-nitro-2-((phenylimino)methyl)phenoxy)hexanoate (3c) Colorless solid ( $\mathrm{MeOH})$ This compound (3c) was prepared from methyl 2-(2-formyl-4-nitrophenoxy)hexanoate (1c) $(1.69 \mathrm{mmol}, 0.50 \mathrm{~g})$ and aniline (2a) $(1.69 \mathrm{mmol}, 0.154 \mathrm{~g})$ according to the general procedure. The product obtained as a colorless solid was purified from methanol. $0.56 \mathrm{~g}(89 \%)$; $\mathrm{mp} 68-71{ }^{\circ} \mathrm{C}$; FTIR (neat) $v_{\max }$ : $3088,2956,1737,1611,1579,1510,1339,1203$, $1072 \mathrm{~cm}^{-1} ;{ }^{1} \mathrm{H}$ NMR $\left(\mathrm{CDCl}_{3}, 400 \mathrm{MHz}\right): \delta=9.09(\mathrm{~d}$, $J=2.9 \mathrm{~Hz}, 1 \mathrm{H}, \mathrm{Ar}), 8.94(\mathrm{~s}, 1 \mathrm{H}, \mathrm{N}=\mathrm{CH}), 8.27$ (dd, $J=2.9,9.1 \mathrm{~Hz}, 1 \mathrm{H}, \mathrm{Ar}), 7.46-7.40$ (m, 2H, Ar), 7.31-7.25 $(\mathrm{m}, 3 \mathrm{H}, A r), 6.86(\mathrm{~d}, J=9.1 \mathrm{~Hz}, 1 \mathrm{H}, \mathrm{Ar}), 4.86(\mathrm{t}$, $J=6.2 \mathrm{~Hz}, 1 \mathrm{H}, \mathrm{CH}), 3.78\left(\mathrm{~s}, 3 \mathrm{H}, \mathrm{OCH}_{3}\right), 2.15-2.00(\mathrm{~m}$, $\left.2 \mathrm{H}, \mathrm{CH}_{2}\right), 1.57-1.46\left(\mathrm{~m}, 2 \mathrm{H}, \mathrm{CH}_{2}\right), 1.46-1.35(\mathrm{~m}, 2 \mathrm{H}$, $\left.\mathrm{CH}_{2}\right), 0.94\left(\mathrm{t}, J=7.2 \mathrm{~Hz}, 3 \mathrm{H}, \mathrm{CH}_{3}\right) ;{ }^{13} \mathrm{C}$ NMR $\left(\mathrm{CDCl}_{3}\right.$, $101 \mathrm{MHz}): \delta=170.7(\mathrm{C}, \mathrm{C}-2), 161.7(\mathrm{CH}, \mathrm{C}-12), 153.5$
(C, C-5), 151.6 (C, C-13), 142.4 (C, C-8), 129.3 (C, C-15, C-17), 127.4 (C, C-7), 126.6 (C, C-9), 126.1 (C, C-10), 124.0 (C, C-16), 121.1 (C, C-14, C-18), 112.5 (C, C-6), 77.4 (CH, C-3), $52.7\left(\mathrm{OCH}_{3}, \mathrm{C}-1\right), 32.2\left(\mathrm{CH}_{2} \mathrm{CH}_{2} \mathrm{CH}_{2} \mathrm{CH}_{3}\right.$, C-4), $27.3\left(\mathrm{CH}_{2} \mathrm{CH}_{2} \mathrm{CH}_{2} \mathrm{CH}_{3}, \mathrm{C}-4\right), 22.2\left(\mathrm{CH}_{2} \mathrm{CH}_{2} \mathrm{CH}_{2}\right.$ $\left.\mathrm{CH}_{3}, \mathrm{C}-4\right), 13.9\left(\mathrm{CH}_{2} \mathrm{CH}_{2} \mathrm{CH}_{2} \mathrm{CH}_{3}, \mathrm{C}-4\right)$; GCMS m/z 370 $[\mathrm{M}]^{+}(51), 327$ (52), 311 (31), 295 (6), 278 (24), 242 (27), 225 (21), 203 (100), 195 (32), 188 (10), 167 (21), 145 (10), 104 (17), 93 (40), 77 (46), 59 (20); Anal. Calcd for $\mathrm{C}_{20} \mathrm{H}_{22} \mathrm{~N}_{2} \mathrm{O}_{5}$ : C, 64.85; H, 5.99; N, 7.56. Found: $\mathrm{C}, 64.80$; $\mathrm{H}, 5.64 ; \mathrm{N}, 7.83$.

Methyl 2-(2-methoxy-4-nitro-6-((phenylimino)methyl)phenoxy)butanoate (3d) Colorless solid (MeOH) This compound (3d) was prepared from methyl 2-(2-formyl6-methoxy-4-nitrophenoxy)butanoate (1d) $(1.68 \mathrm{mmol}$, $0.50 \mathrm{~g})$ and aniline $(\mathbf{2 a})(1.68 \mathrm{mmol}, 0.153 \mathrm{~g})$ according to the general procedure. The product obtained as a colorless solid was purified from methanol. $0.53 \mathrm{~g}$ (85\%); mp 124-129 ${ }^{\circ} \mathrm{C}$; FTIR (neat) $v_{\max }: 3088,2977,1746,1619$, 1577, 1518, 1341, 1202, $1085 \mathrm{~cm}^{-1}$; ${ }^{1} \mathrm{H}$ NMR $\left(\mathrm{CDCl}_{3}\right.$, $400 \mathrm{MHz}): \delta=9.09$ (s, $1 \mathrm{H}, \mathrm{N}=\mathrm{CH}), 8.74(\mathrm{~d}, J=2.9 \mathrm{~Hz}$, $1 \mathrm{H}, \mathrm{Ar}), 7.83(\mathrm{~d}, J=2.9 \mathrm{~Hz}, 1 \mathrm{H}, \mathrm{Ar}), 7.46-7.39(\mathrm{~m}, 2 \mathrm{H}$, Ar), 7.33-7.24 (m, 3H, Ar), $5.13(\mathrm{dd}, J=5.4,6.9,1 \mathrm{H}$, $\mathrm{CH}), 3.96\left(\mathrm{~s}, 3 \mathrm{H}, \mathrm{OCH}_{3}\right), 3.69\left(\mathrm{~s}, 3 \mathrm{H}, \mathrm{OCH}_{3}\right), 2.13-1.97$ $\left(\mathrm{m}, 2 \mathrm{H}, \mathrm{CH}_{2}\right), 1.11\left(\mathrm{t}, J=7.6 \mathrm{~Hz}, 3 \mathrm{H}, \mathrm{CH}_{3}\right) ;{ }^{13} \mathrm{C} \mathrm{NMR}$ $\left(\mathrm{CDCl}_{3}, 101 \mathrm{MHz}\right): \delta=171.4(\mathrm{C}, \mathrm{C}-2), 155.0(\mathrm{C}, \mathrm{C}-12)$, 152.0 (C, C-5), 151.7 (C, C-6), 151.6 (C, C-13), 143.7 (C, C-8), 130.2 (C, C-10), 129.2 (C, C-15, C-17), 126.5 (C, C-16), 121.2 (C, C-14, C-18), 115.6 (C, C-9), 108.9 (C, $\mathrm{C}-7), 81.4(\mathrm{CH}, \mathrm{C}-3), 56.4\left(\mathrm{OCH}_{3}, \mathrm{C}-11\right), 52.1\left(\mathrm{OCH}_{3}\right.$, C-1), $26.5\left(\mathrm{CH}_{2} \mathrm{CH}_{3}, \mathrm{C}-4\right), 9.4\left(\mathrm{CH}_{2} \mathrm{CH}_{3}, \mathrm{C}-4\right)$; GCMS $\mathrm{m} / \mathrm{z}$ $372[\mathrm{M}]^{+}(61), 357$ (11), 313 (34), 280 (37), 280 (37), 271 (15), 255 (22), 252 (7), 236 (12), 225 (22), 210 (8), 205 (100), 196 (12), 190 (29), 175 (45), 154 (25), 127 (6), 104 (16), 93 (19), 77 (38), 59 (23), 51 (6); Anal. Calcd for $\mathrm{C}_{19} \mathrm{H}_{20} \mathrm{~N}_{2} \mathrm{O}_{6}$ : C, 61.28; H, 5.41; N, 7.52. Found: C, 61.24; $\mathrm{H}, 5.39 ; \mathrm{N}, 7.55$.

Methyl 2-(2-methoxy-4-nitro-6-((phenylimino)methyl)phenoxy)pentanoate (3e) Colorless solid $(\mathrm{MeOH})$ This compound (3e) was prepared from methyl 2-(2-formyl-6methoxy-4-nitrophenoxy)pentanoate (1e) $\quad(1.61 \mathrm{mmol}$, $0.50 \mathrm{~g})$ and aniline $(\mathbf{2 a})(1.62 \mathrm{mmol}, 0.147 \mathrm{~g})$ according to the general procedure. The product obtained as a colorless solid was purified from methanol. $0.53 \mathrm{~g}(85 \%)$; mp 126-128 ${ }^{\circ} \mathrm{C}$; FTIR (neat) $v_{\max }$ : 3086, 2961, 1745, 1623, $1575,1517,1328,1205,1071 \mathrm{~cm}^{-1} ;{ }^{1} \mathrm{H}$ NMR $\left(\mathrm{CDCl}_{3}\right.$, $400 \mathrm{MHz}): \delta=9.08(\mathrm{~s}, 1 \mathrm{H}, \mathrm{N}=\mathrm{CH}), 8.74(\mathrm{~d}, J=2.7 \mathrm{~Hz}$, $1 \mathrm{H}, \mathrm{Ar}), 7.83(\mathrm{~d}, J=2.7 \mathrm{~Hz}, 1 \mathrm{H}, \mathrm{Ar}), 7.45-7.39(\mathrm{~m}, 2 \mathrm{H}$, Ar), 7.45-7.39 (m, 3H, Ar), 5.19 (dd, $J=5.6,6.7 \mathrm{~Hz}, 1 \mathrm{H}$, $\mathrm{CH}), 3.96\left(\mathrm{~s}, 3 \mathrm{H}, \mathrm{OCH}_{3}\right), 3.67\left(\mathrm{~s}, 3 \mathrm{H}, \mathrm{OCH}_{3}\right), 2.06-1.91$ $\left(\mathrm{m}, 2 \mathrm{H}, \mathrm{CH}_{2}\right), \quad 1.65-1.51\left(\mathrm{~m}, 2 \mathrm{H}, \mathrm{CH}_{2}\right), 1.00(\mathrm{t}$, $\left.J=7.4 \mathrm{~Hz}, 3 \mathrm{H}, \mathrm{CH}_{3}\right) ;{ }^{13} \mathrm{C}$ NMR $\left(\mathrm{CDCl}_{3}, 101 \mathrm{MHz}\right)$ : 
$\delta=171.6(\mathrm{C}, \mathrm{C}-2), 155.1$ (C, C-12), 152.0 (C, C-5), 151.7 (C, C-6), 151.6 (C, C-13), 143.7 (C, C-8), 130.2 (C, C-10), 129.2 (C, C-15, C-17), 126.5 (C, C-16), 121.2 (C, C-14, C-18), 115.6 (C, C-9), 108.8 (C, C-7), 80.1 (CH, C-3), 56.4 $\left(\mathrm{OCH}_{3}, \mathrm{C}-11\right), 52.1\left(\mathrm{OCH}_{3}, \mathrm{C}-1\right), 35.2\left(\mathrm{CH}_{2} \mathrm{CH}_{2} \mathrm{CH}_{3}\right.$, C-4), $18.3\left(\mathrm{CH}_{2} \mathrm{CH}_{2} \mathrm{CH}_{3}, \mathrm{C}-4\right), 13.7\left(\mathrm{CH}_{2} \mathrm{CH}_{2} \mathrm{CH}_{3}, \mathrm{C}-4\right)$; GCMS m/z $386[\mathrm{M}]^{+}$(52), 357 (34), 327 (23), 297 (8), 294 (36), 271 (16), 255 (23), 235 (13), 219 (100), 204 (25), 189 (36), 175 (15), 154 (27), 127 (7), 104 (18), 93 (32), 77 (42), 59 (20); Anal. Calcd for $\mathrm{C}_{20} \mathrm{H}_{22} \mathrm{~N}_{2} \mathrm{O}_{6}: \mathrm{C}, 62.17 ; \mathrm{H}, 5.74 ; \mathrm{N}$, 7.25. Found: C, 62.47; H, 5.70; N, 7.30.

Methyl 2-(2-methoxy-4-nitro-6-((phenylimino)methyl)phenoxy)hexanoate (3f) Colorless solid (MeOH) This compound (3f) was prepared from methyl 2-(2-formyl-6methoxy-4-nitrophenoxy)hexanoate (1f) $\quad(1.54 \mathrm{mmol}$, $0.50 \mathrm{~g})$ and aniline (2a) $(1.54 \mathrm{mmol}, 0.14 \mathrm{~g})$ according to the general procedure. The product obtained as a colorless solid was purified from methanol. $0.48 \mathrm{~g}$ (78 \%); mp 82-91 ${ }^{\circ} \mathrm{C}$; FTIR (neat) $v_{\max }: 3090,2953,1744,1619,1575$, 1525, 1322, 1200, $1091 \mathrm{~cm}^{-1} ;{ }^{1} \mathrm{H}$ NMR $\left(\mathrm{CDCl}_{3}\right.$, $400 \mathrm{MHz}): \delta=9.08(\mathrm{~s}, 1 \mathrm{H}, \mathrm{N}=\mathrm{CH}), 8.74(\mathrm{~d}, J=2.7 \mathrm{~Hz}$, $1 \mathrm{H}, \mathrm{Ar}), 7.83(\mathrm{~d}, J=2.7 \mathrm{~Hz}, 1 \mathrm{H}, \mathrm{Ar}), 7.46-7.39(\mathrm{~m}, 2 \mathrm{H}$, Ar), 7.33-7.24 (m, 3H, Ar), $5.17(\mathrm{t}, J=6.1 \mathrm{~Hz}, 1 \mathrm{H}, \mathrm{CH})$, $3.96\left(\mathrm{~s}, 3 \mathrm{H}, \mathrm{OCH}_{3}\right), 3.68\left(\mathrm{~s}, 3 \mathrm{H}, \mathrm{OCH}_{3}\right), 2.06-1.96(\mathrm{~m}, 2 \mathrm{H}$, $\left.\mathrm{CH}_{2}\right), 1.58-1.46\left(\mathrm{~m}, 2 \mathrm{H}, \mathrm{CH}_{2}\right), 1.45-1.34\left(\mathrm{~m}, 2 \mathrm{H}, \mathrm{CH}_{2}\right)$, $0.93\left(\mathrm{t}, \quad J=7.3 \mathrm{~Hz}, 3 \mathrm{H}, \mathrm{CH}_{3}\right) ;{ }^{13} \mathrm{C} \quad \mathrm{NMR}\left(\mathrm{CDCl}_{3}\right.$, $101 \mathrm{MHz}): \delta=171.6$ (C, C-2), 155.2 (C, C-12), 152.0 (C, C-5), 151.7 (C, C-6), 151.6 (C, C-13), 143.7 (C, C-8), 130.2 (C, C-10), 129.2 (C, C-15, C-17), 126.5 (C, C-16), 121.2 (C, C-14, C-18), 115.5 (C, C-9), 108.8 (C, C-7), 80.3 $(\mathrm{CH}, \mathrm{C}-3), 56.4\left(\mathrm{OCH}_{3}, \mathrm{C}-11\right), 52.1\left(\mathrm{OCH}_{3}, \mathrm{C}-1\right), 32.9$ $\left(\mathrm{CH}_{2} \mathrm{CH}_{2} \mathrm{CH}_{2} \mathrm{CH}_{3}, \mathrm{C}-4\right), 27.1 \quad\left(\mathrm{CH}_{2} \mathrm{CH}_{2} \mathrm{CH}_{2} \mathrm{CH}_{3}, \mathrm{C}-4\right)$, $22.4\left(\mathrm{CH}_{2} \mathrm{CH}_{2} \mathrm{CH}_{2} \mathrm{CH}_{3}, \mathrm{C}-4\right), 13.9 \quad\left(\mathrm{CH}_{2} \mathrm{CH}_{2} \mathrm{CH}_{2} \mathrm{CH}_{3}\right.$, C-4); GCMS m/z $400\left[\mathrm{M}^{+}\right.$(67), 369 (5), 357 (43), 341 (29), 308 (43), 297 (7), 276 (6), 271 (19), 255 (25), 248 (10), 233 (100), 226 (9), 210 (8), 203 (36), 196 (14), 180 (14), 154 (27), 127 (6), 104 (16), 93 (33), 77 (36), 69 (14); Anal. Calcd for $\mathrm{C}_{21} \mathrm{H}_{24} \mathrm{~N}_{2} \mathrm{O}_{6}$ : C, 62.99; H, 6.04; N, 7.00. Found: C, 63.10; H, 6.01; N, 6.97.

Ethyl 2-(2-methoxy-4-nitro-6-((phenylimino)methyl)phenoxy)acetate (3g) Colorless solid $(\mathrm{MeOH})$ This compound (3 $\mathbf{g})$ was prepared from ethyl 2-(2-formyl-4-nitrophenoxy)acetate (1 g) $(1.77 \mathrm{mmol}, 0.50 \mathrm{~g})$ and aniline (2a) $(1.77 \mathrm{mmol}, 0.161 \mathrm{~g})$ according to the general procedure. The product obtained as an aquamarine solid was purified from methanol. $0.57 \mathrm{~g}(90 \%)$; mp $98-101{ }^{\circ} \mathrm{C}$; FTIR (neat) $v_{\text {max }}: 3107,2944,1756,1617,1577,1516,1337$, $1199 \mathrm{~cm}^{-1} ;{ }^{1} \mathrm{H}$ NMR $\left(\mathrm{CDCl}_{3}, 400 \mathrm{MHz}\right): \delta=9.10(\mathrm{~s}, 1 \mathrm{H}$, $\mathrm{N}=\mathrm{CH}), 8.74(\mathrm{~d}, J=2.6 \mathrm{~Hz}, 1 \mathrm{H}, \mathrm{Ar}), 7.85(\mathrm{~d}, J=2.6 \mathrm{~Hz}$, $1 \mathrm{H}, \mathrm{Ar}), 7.45-7.38$ (m, 2H, Ar), 7.34-7.23 (m, 3H, Ar), $4.91\left(\mathrm{~s}, 2 \mathrm{H}, \mathrm{CH}_{2}\right), 4.22\left(\mathrm{q}, J=7.1 \mathrm{~Hz}, 2 \mathrm{H}, \mathrm{OCH}_{2}\right), 3.99$ (s, $\left.3 \mathrm{H}, \mathrm{OCH}_{3}\right), 1.24\left(\mathrm{t}, J=7.1 \mathrm{~Hz}, 3 \mathrm{H}, \mathrm{CH}_{3}\right) ;{ }^{13} \mathrm{C} \mathrm{NMR}$
$\left(\mathrm{CDCl}_{3}, 101 \mathrm{MHz}\right): \delta=168.8(\mathrm{C}, \mathrm{C}-2), 154.8(\mathrm{CH}, \mathrm{C}-12)$, 152.2 (C, C-5), 151.8 (C, C-6), 151.4 (C, C-13), 144.0 (C, C-8), 130.1 (C, C-10), 129.2 (C, C-15, C-17), 126.6 (C, C-16), 121.3 (C, C-14, C-18), 115.4 (C, C-9), 108.9 (C, $\mathrm{C}-7), 69.5\left(\mathrm{CH}_{2}, \mathrm{C}-3\right), 61.4\left(\mathrm{OCH}_{2} \mathrm{CH}_{3}, \mathrm{C}-1\right), 56.5\left(\mathrm{OCH}_{3}\right.$, $\mathrm{C}-11), 14.1\left(\mathrm{OCH}_{2} \mathrm{CH}_{3}, \mathrm{C}-1\right) ; \mathrm{GCMS} \mathrm{m} / z 358[\mathrm{M}]^{+}(19)$, 329 (3), 285 (52), 255 (6), 239 (16), 225 (6), 210 (5), 194 (22), 179 (7), 167 (6), 148 (31), 104 (11), 93 (100), 77 (28), 61 (5). Anal. Calcd for $\mathrm{C}_{19} \mathrm{H}_{20} \mathrm{~N}_{2} \mathrm{O}_{6}$ : C, 61.28; H, 5.41; N, 7.52. Found: C, $61.32 ; \mathrm{H}, 5.52 ; \mathrm{N}, 7.50$.

Methyl 2-(2-((4-methoxyphenylimino)methyl)-4-nitrophenoxy)butanoate (3h) Aquamarine solid ( $\mathrm{MeOH})$ This compound $(\mathbf{3} \mathbf{h})$ was prepared from methyl 2-(2-formyl-4nitrophenoxy)butanoate (1a) $(1.69 \mathrm{mmol}, 0.45 \mathrm{~g})$ and $p$ methoxyaniline (2b) (1.69 mmol, $0.205 \mathrm{~g})$ according to the general procedure. The product obtained as an aquamarine solid was purified from methanol. $0.52 \mathrm{~g}(83 \%)$; mp 108.5-110.5 ${ }^{\circ} \mathrm{C}$; FTIR (neat) $v_{\max }: 3090,2932,1737,1615$, 1596, 1510, 1342, 1214, $1075 \mathrm{~cm}^{-1}$; ${ }^{1} \mathrm{H}$ NMR $\left(\mathrm{CDCl}_{3}\right.$, $400 \mathrm{MHz}): \delta=9.08(\mathrm{~d}, J=2.9 \mathrm{~Hz}, 1 \mathrm{H}, \mathrm{Ar}), 8.96(\mathrm{~s}, 1 \mathrm{H}$, $\mathrm{N}=\mathrm{CH}), 8.24(\mathrm{dd}, J=2.9,9.1 \mathrm{~Hz}, 1 \mathrm{H}, \mathrm{Ar}), 7.33-7.28(\mathrm{~m}$, 2H, Ar), 6.99-6.94 (m, 2H, Ar), $6.84(\mathrm{~d}, J=9.1 \mathrm{~Hz}, 1 \mathrm{H}$, $\mathrm{Ar}), 4.82(\mathrm{t}, J=6.0 \mathrm{~Hz}, 1 \mathrm{H}, \mathrm{CH}), 3.85\left(\mathrm{~s}, 3 \mathrm{H}, \mathrm{OCH}_{3}\right)$, $3.78\left(\mathrm{~s}, 3 \mathrm{H}, \mathrm{OCH}_{3}\right), 2.12\left(\mathrm{p}, J=7.4 \mathrm{~Hz}, 2 \mathrm{H}, \mathrm{CH}_{2}\right), 1.11(\mathrm{t}$, $\left.J=7.4 \mathrm{~Hz}, 3 \mathrm{H}, \mathrm{CH}_{3}\right) ;{ }^{13} \mathrm{C}$ NMR $\left(\mathrm{CDCl}_{3}, 101 \mathrm{MHz}\right)$ : $\delta=170.5$ (C, C-2), 161.5 (C, C-12), 158.8 (C, C-5), 151.0 (C, C-16), 144.3 (C, C-13), 142.3 (C, C-8), 127.0 (C, C-7), 126.4 (C, C-10), 123.6 (C, C-9), 122.6 (C, C-14, C-18), 114.4 (C, C-15, C-17), 112.5 (C, C-6), 78.3 (CH, C-3), 55.5 $\left(\mathrm{OCH}_{3}, \mathrm{C}-19\right), 52.6\left(\mathrm{OCH}_{3}, \mathrm{C}-1\right), 26.0\left(\mathrm{CH}_{2} \mathrm{CH}_{3}, \mathrm{C}-4\right), 9.6$ $\left(\mathrm{CH}_{2} \mathrm{CH}_{3}, \mathrm{C}-4\right)$; GCMS m/z $372[\mathrm{M}]^{+}$(100), 313 (16), 271 (9), 257 (12), 241 (11), 225 (16), 190 (5), 175 (54), 160 (7), 145 (14), 134 (7), 122 (60), 108 (7), 92 (10), 77 (10), 59 (14); Anal. Calcd for $\mathrm{C}_{19} \mathrm{H}_{20} \mathrm{~N}_{2} \mathrm{O}_{6}: \mathrm{C}, 61.28 ; \mathrm{H}, 5.41 ; \mathrm{N}$, 7.52. Found: C, 61.32; H, 5.52; N, 7.50.

Methyl 2-(2-((4-methoxyphenylimino)methyl)-4-nitrophenoxy)hexanoate (3i) Pale yellow solid $(\mathrm{MeOH})$ This compound (3i) was prepared from methyl 2-(2-formyl-4nitrophenoxy)hexanoate (1c) $(1.69 \mathrm{mmol}, 0.50 \mathrm{~g})$ and $p$ methoxyaniline (2b) $(1.69 \mathrm{mmol}, 0.208 \mathrm{~g})$ according to the general procedure. The product obtained as a pale yellow solid was purified from methanol. $0.49 \mathrm{~g}$ (72\%); mp 102-104 ${ }^{\circ} \mathrm{C}$; FTIR (neat) $v_{\max }: 3090,2927,1739,1616$, 1592, 1516, 1333, 1198, $1080 \mathrm{~cm}^{-1} ;{ }^{1} \mathrm{H}$ NMR $\left(\mathrm{CDCl}_{3}\right.$, $400 \mathrm{MHz}): \delta=9.08$ (d, $J=2.9 \mathrm{~Hz}, 1 \mathrm{H}, \mathrm{Ar}), 8.95$ (s, $1 \mathrm{H}$, $\mathrm{N}=\mathrm{CH}), 8.24(\mathrm{dd}, J=2.9,9.2 \mathrm{~Hz}, 1 \mathrm{H}, \mathrm{Ar}), 7.33-7.28(\mathrm{~m}$, 2H, Ar), 6.99-6.93 (m, 2H, Ar), $6.84(\mathrm{~d}, J=9.2 \mathrm{~Hz}, 1 \mathrm{H}$, $\mathrm{Ar}$ ), 4.85 (undivided dd, $J=5.6,6.8 \mathrm{~Hz}, 1 \mathrm{H}, \mathrm{CH}$ ), 3.85 (s, $\left.3 \mathrm{H}, \mathrm{OCH}_{3}\right), 3.78\left(\mathrm{~s}, 3 \mathrm{H}, \mathrm{OCH}_{3}\right), 2.12-2.02\left(\mathrm{~m}, 2 \mathrm{H}, \mathrm{CH}_{2}\right)$, 1.57-1.47 (m, 2H, $\left.\mathrm{CH}_{2}\right), 1.47-1.36\left(\mathrm{~m}, 2 \mathrm{H}, \mathrm{CH}_{2}\right), 0.94(\mathrm{t}$, $\left.J=7.2 \mathrm{~Hz}, 3 \mathrm{H}, \mathrm{CH}_{3}\right) ;{ }^{13} \mathrm{C}$ NMR $\left(\mathrm{CDCl}_{3}, 101 \mathrm{MHz}\right)$ : $\delta=170.8$ (C, C-2), 161.5 (C, C-12), 158.8 (C, C-5), 151.1 
(C, C-16), 144.3 (C, C-13), 142.3 (C, C-8), 127.0 (C, C-7), 126.4 (C, C-10), 123.7 (C, C-9), 122.6 (C, C-14, C-18), 114.4 (C, C-15, C-17), 112.4 (C, C-6), 77.4 (CH, C-3), 55.5 $\left(\mathrm{OCH}_{3}, \mathrm{C}-19\right), 52.6\left(\mathrm{OCH}_{3}, \mathrm{C}-1\right), 32.2\left(\mathrm{CH}_{2} \mathrm{CH}_{2} \mathrm{CH}_{2} \mathrm{CH}_{3}\right.$, C-4), $27.3\left(\mathrm{CH}_{2} \mathrm{CH}_{2} \mathrm{CH}_{2} \mathrm{CH}_{3}, \mathrm{C}-4\right), 22.2\left(\mathrm{CH}_{2} \mathrm{CH}_{2}-\mathrm{CH}_{2}\right.$ $\left.\mathrm{CH}_{3}, \mathrm{C}-4\right), 13.9\left(\mathrm{CH}_{2} \mathrm{CH}_{2} \mathrm{CH}_{2} \mathrm{CH}_{3}, \mathrm{C}-4\right)$; GCMS $\mathrm{m} / z, 400$ $\left[\mathrm{M}^{+}(100), 357\right.$ (12), 341 (14), 297 (6), 272 (15), 257 (13), 241 (9), 225 (16), 203 (52), 182 (6), 173 (6), 154 (7), 145 (6), 134 (6), 122 (40), 108 (6), 92 (8), 77 (8), 59 (8); Anal. Calcd for $\mathrm{C}_{21} \mathrm{H}_{24} \mathrm{~N}_{2} \mathrm{O}_{6}$ : C, 62.99; H, 6.04; N, 7.00. Found: C, 62.96; H, 6.14; N, 7.02.

Methyl 2-(2-((4-methoxyphenylimino)methyl)-6-methoxy-4nitrophenoxy)butanoate (3j) Beige solid $(\mathrm{MeOH})$ This compound (3j) was prepared from methyl 2-(2-formyl6-methoxy-4-nitrophenoxy)butanoate (1d) $\quad(3.37 \mathrm{mmol}$, $1.00 \mathrm{~g})$ and $p$-methoxyaniline (2b) $(3.37 \mathrm{mmol}, 0.415 \mathrm{~g})$ according to the general procedure. The product obtained as a beige solid was purified from methanol. $1.20 \mathrm{~g} \mathrm{(89 \% );}$ mp $116-117{ }^{\circ} \mathrm{C}$; FTIR (neat) $v_{\max }: 3099,2948,1746,1619$, $1577,1518,1341,1202,1091 \mathrm{~cm}^{-1} ;{ }^{1} \mathrm{H}$ NMR $\left(\mathrm{CDCl}_{3}\right.$, $400 \mathrm{MHz}): \delta=9.10(\mathrm{~s}, 1 \mathrm{H}, \mathrm{N}=\mathrm{CH}), 8.73(\mathrm{~d}, J=2.6 \mathrm{~Hz}$, $1 \mathrm{H}, \mathrm{Ar}), 7.80(\mathrm{~d}, J=2.6 \mathrm{~Hz}, 1 \mathrm{H}, \mathrm{Ar}), 7.37-7.32(\mathrm{~m}, 2 \mathrm{H}$, Ar), 7.00-6.93 (m, 2H, Ar), $5.10(\mathrm{dd} J=5.6,6.4 \mathrm{~Hz}, 1 \mathrm{H}$, $\mathrm{CH}), 3.95\left(\mathrm{~s}, 3 \mathrm{H}, \mathrm{OCH}_{3}\right), 3.85\left(\mathrm{~s}, 3 \mathrm{H}, \mathrm{OCH}_{3}\right), 3.69(\mathrm{~s}, 3 \mathrm{H}$, $\left.\mathrm{OCH}_{3}\right), 2.14-1.98\left(\mathrm{~m}, 2 \mathrm{H}, \mathrm{CH}_{2}\right), 1.11(\mathrm{t}, J=7.5 \mathrm{~Hz}, 3 \mathrm{H}$, $\left.\mathrm{CH}_{3}\right) ;{ }^{13} \mathrm{C} \mathrm{NMR}\left(\mathrm{CDCl}_{3}, 101 \mathrm{MHz}\right): \delta=171.4(\mathrm{C}, \mathrm{C}-2)$, 158.8 (C, C-12), 152.6 (C, C-16), 151.7 (C, C-5, C-6), 144.3 (C, C-13), 143.7 (C, C-8), 130.5 (C, C-10), 122.7 (C, C-14, C-18), 115.4 (C, C-9), 114.4 (C, C-15, C-17), 108.5 (C, C-7), $81.4(\mathrm{CH}, \mathrm{C}-3), 56.4\left(\mathrm{OCH}_{3}, \mathrm{C}-11\right), 55.5\left(\mathrm{OCH}_{3}\right.$, $\mathrm{C}-19), 52.0\left(\mathrm{OCH}_{3}, \mathrm{C}-1\right), 26.5\left(\mathrm{CH}_{2} \mathrm{CH}_{3}, \mathrm{C}-4\right), 9.4\left(\mathrm{CH}_{2-}\right.$ $\left.\mathrm{CH}_{3}, \mathrm{C}-4\right)$; GCMS $m / 2,402[\mathrm{M}]^{+}(100), 343$ (12), 301 (11), 281 (7), 271 (11), 255 (20), 236 (7), 221 (12), 205 (88), 190 (25), 175 (40), 162 (7), 134 (14), 122 (48), 108 (10), 92 (12), 77 (15), 59 (22); Anal. Calcd for $\mathrm{C}_{20} \mathrm{H}_{22} \mathrm{~N}_{2} \mathrm{O}_{7}$ : C, 59.70; H, 5.51; N, 6.96. Found: C, 59.82; H, 5.62; N, 6.76.

Methyl 2-(2-((4-methoxyphenylimino)methyl)-6-methoxy-4nitrophenoxy)pentanoate (3k) Pale yellow solid $(\mathrm{MeOH})$ This compound (3k) was prepared from methyl 2-(2-formyl-6-methoxy-4-nitrophenoxy)pentanoate

(1e) $(3.22 \mathrm{mmol}, \quad 1.00 \mathrm{~g})$ and $p$-methoxyaniline (2b) (3.22 mmol, $0.396 \mathrm{~g}$ ) according to the general procedure. The product obtained as a pale yellow solid was purified from methanol. $1.20 \mathrm{~g}$ (90\%); mp 105-106 ${ }^{\circ} \mathrm{C}$; FTIR (neat) $v_{\max }: 3099,2952,1736,1616,1572,1522,1342$, $1204,1087 \mathrm{~cm}^{-1} ;{ }^{1} \mathrm{H} \mathrm{NMR}\left(\mathrm{CDCl}_{3}, 400 \mathrm{MHz}\right): \delta=9.10$ $(\mathrm{s}, 1 \mathrm{H}, \mathrm{N}=\mathrm{CH}), 8.73(\mathrm{~d}, J=2.5 \mathrm{~Hz}, 1 \mathrm{H}, \mathrm{Ar}), 7.80(\mathrm{~d}$, $J=2.6 \mathrm{~Hz}, 1 \mathrm{H}, \mathrm{Ar}), 7.40-7.31$ (m, 2H, Ar), 7.00-6.92 (m, $2 \mathrm{H}, \mathrm{Ar}$ ), 5.16 (undivided dd, $J=5.6,6.8 \mathrm{~Hz}, 1 \mathrm{H}, \mathrm{CH}$ ), $3.95\left(\mathrm{~s}, 3 \mathrm{H}, \mathrm{OCH}_{3}\right), 3.85\left(\mathrm{~s}, 3 \mathrm{H}, \mathrm{OCH}_{3}\right), 3.67(\mathrm{~s}, 3 \mathrm{H}$, $\left.\mathrm{OCH}_{3}\right), 2.09-1.90\left(\mathrm{~m}, 2 \mathrm{H}, \mathrm{CH}_{2}\right), 1.71-1.46\left(\mathrm{~m}, 2 \mathrm{H}, \mathrm{CH}_{2}\right)$, $1.00\left(\mathrm{t}, J=7.4 \mathrm{~Hz}, 3 \mathrm{H}, \mathrm{CH}_{3}\right) ;{ }^{13} \mathrm{C} \quad \mathrm{NMR}\left(\mathrm{CDCl}_{3}\right.$,
$101 \mathrm{MHz}): \delta=171.7$ (C, C-2), 158.8 (C, C-12), 152.7 (C, C-16), 151.7 (C, C-5, C-6), 144.3 (C, C-13), 143.7 (C, C-8), 130.5 (C, C-10), 122.7 (C, C-14, C-18), 115.3 (C, C-9), 114.4 (C, C-15, C-17), 108.4 (C, C-7), 80.1 (CH, $\mathrm{C}-3), 56.3\left(\mathrm{OCH}_{3}, \mathrm{C}-11\right), 55.5\left(\mathrm{OCH}_{3}, \mathrm{C}-19\right), 52.1\left(\mathrm{OCH}_{3}\right.$, $\mathrm{C}-1), 35.2\left(\mathrm{CH}_{2} \mathrm{CH}_{2} \mathrm{CH}_{3}, \mathrm{C}-4\right), 18.3\left(\mathrm{CH}_{2} \mathrm{CH}_{2} \mathrm{CH}_{3}, \mathrm{C}-4\right)$, $13.8\left(\mathrm{CH}_{2} \mathrm{CH}_{2} \mathrm{CH}_{3}, \mathrm{C}-4\right)$; GCMS m/z $416[\mathrm{M}]^{+}(100), 387$ (8), 357 (11), 301 (10), 285 (6), 271 (5), 255 (17), 235 (8), 219 (61), 204 (15), 189 (22), 184 (6), 175 (9), 134 (8), 122 (21), 108 (6), 92 (6), 77 (7), 59 (8); Anal. Calcd for $\mathrm{C}_{21} \mathrm{H}_{24} \mathrm{~N}_{2} \mathrm{O}_{7}$ : C, 60.57; H, 5.81; N, 6.73. Found: C, 60.65; $\mathrm{H}, 5.72 ; \mathrm{N}, 6.73$.

Methyl 2-(2-((4-methoxyphenylimino)methyl)-6-methoxy-4nitrophenoxy)hexanoate (3l) Aquamarine solid ( $\mathrm{MeOH})$ This compound (31) was prepared from methyl 2-(2-formyl-6-methoxy-4-nitrophenoxy)hexanoate (1f) $(3.08 \mathrm{mmol}, 1.00 \mathrm{~g})$ and $p$-methoxyaniline $(\mathbf{2 b})(3.08 \mathrm{mmol}$, $0.379 \mathrm{~g}$ ) according to the general procedure. The product obtained as an aquamarine solid was purified from methanol. $1.32 \mathrm{~g}$ (76\%); mp $116-119{ }^{\circ} \mathrm{C}$; FTIR (neat) $v_{\max }: 3102,2955,1739,1616,1592,1516,1333,1198$, $1092 \mathrm{~cm}^{-1} ;{ }^{1} \mathrm{H} \mathrm{NMR}\left(\mathrm{CDCl}_{3}, 400 \mathrm{MHz}\right): \delta=9.10(\mathrm{~s}, 1 \mathrm{H}$, $\mathrm{N}=\mathrm{CH}), 8.72(\mathrm{~d}, J=2.3 \mathrm{~Hz}, 1 \mathrm{H}, \mathrm{Ar}), 7.80(\mathrm{~d}, J=2.3 \mathrm{~Hz}$, 1H, Ar), 7.37-7.32 (m, 2H, Ar), 6.99-6.93 (m, 2H, Ar), $5.13(\mathrm{t}, J=6.0 \mathrm{~Hz}, 1 \mathrm{H}, \mathrm{CH}), 3.95\left(\mathrm{~s}, 3 \mathrm{H}, \mathrm{OCH}_{3}\right), 3.85(\mathrm{~s}$, $\left.3 \mathrm{H}, \mathrm{OCH}_{3}\right), 3.68\left(\mathrm{~s}, 3 \mathrm{H}, \mathrm{OCH}_{3}\right), 2.00(\mathrm{p}, J=7.0 \mathrm{~Hz}, 2 \mathrm{H}$, $\left.\mathrm{CH}_{2}\right), 1.59-1.46\left(\mathrm{~m}, 2 \mathrm{H}, \mathrm{CH}_{2}\right), 1.45-1.34\left(\mathrm{~m}, 2 \mathrm{H}, \mathrm{CH}_{2}\right)$, $0.93\left(\mathrm{t}, \quad J=7.2 \mathrm{~Hz}, 3 \mathrm{H}, \mathrm{CH}_{3}\right) ;{ }^{13} \mathrm{C} \quad \mathrm{NMR}\left(\mathrm{CDCl}_{3}\right.$, $101 \mathrm{MHz}): \delta=171.6$ (C, C-2), 158.8 (C, C-12), 152.7 (C, C-16), 151.7 (C, C-5), 151.6 (C, C-6), 144.3 (C, C-13), 143.7 (C, C-8), 130.5 (C, C-10), 122.7 (C, C-14, C-18), 115.3 (C, C-9), 114.4 (C, C-15, C-17), 108.5 (C, C-7), 80.4 (CH, C-3), $56.3\left(\mathrm{OCH}_{3}, \mathrm{C}-11\right), 55.5\left(\mathrm{OCH}_{3}, \mathrm{C}-19\right), 52.1$ $\left(\mathrm{OCH}_{3}, \mathrm{C}-1\right), 32.9\left(\mathrm{CH}_{2} \mathrm{CH}_{2} \mathrm{CH}_{2} \mathrm{CH}_{3}, \mathrm{C}-4\right), 27.1\left(\mathrm{CH}_{2-}\right.$ $\left.\mathrm{CH}_{2} \mathrm{CH}_{2} \mathrm{CH}_{3}, \mathrm{C}-4\right), 22.4\left(\mathrm{CH}_{2} \mathrm{CH}_{2} \mathrm{CH}_{2} \mathrm{CH}_{3}, \mathrm{C}-4\right), 13.9$ $\left(\mathrm{CH}_{2} \mathrm{CH}_{2} \mathrm{CH}_{2} \mathrm{CH}_{3}, \mathrm{C}-4\right)$; GCMS $m / z 430[\mathrm{M}]^{+}$(100), 387 (8), 371 (11), 301 (10), 285 (6), 271 (5), 262 (2), 255 (17), 248 (5), 233 (59), 218 (12), 206 (7), 203 (23), 184 (6), 175 (7), 134 (8), 122 (20), 108 (6), 92 (6), 77 (7), 59 (7). Anal. Calcd for $\mathrm{C}_{22} \mathrm{H}_{26} \mathrm{~N}_{2} \mathrm{O}_{7}$ : C, 61.39; H, 6.09; N, 6.51. Found: C, 61.45; H, 6.20; N, 6.75 .

\section{General procedure for the synthesis of methyl 2-(4- nitro-2-((phenylamino)methyl)phenoxy)alkanoate (4a-f)}

To a mixture of methyl 2-(4-nitro-2-((phenylimino) methyl)phenoxy)alkanoate (3a-f) $(0.58 \mathrm{mmol})$ in $1,2-$ dichloroethane $(10 \mathrm{~mL})$, sodium triacetoxyborohydride $(0.88 \mathrm{mmol})$ and acetic acid $(0.1 \mathrm{~mL})$ were added. The mixture was stirred magnetically at room temperature for $4 \mathrm{~h}$. Then, the mixture was quenched with an aqueous 
solution of sodium carbonate $5 \%\left(\mathrm{NaHCO}_{3}\right)$. The DCE layer was separated and dried $\left(\mathrm{MgSO}_{4}\right)$. The solvent was removed under reduced pressure to give the crude product. The resultant organic layer was dried, evaporated, and residue was recrystallized from hot methanol, to yield $\mathbf{4 a}-\mathbf{f}$.

Methyl 2-(4-nitro-2-((phenylamino)methyl)phenoxy)butanoate (4a) Yellow solid (MeOH) This compound (4a) was prepared from methyl 2-(4-nitro-2-((phenylimino)methyl)phenoxy)butanoate (3a) $(0.58 \mathrm{mmol}, 0.20 \mathrm{~g})$ and sodium triacetoxyborohydride $(0.88 \mathrm{mmol}, 0.186 \mathrm{~g})$ according to the general procedure. The product obtained as a yellow solid was purified from methanol. $0.12 \mathrm{~g}$ (60 \%); mp 106-108 ${ }^{\circ} \mathrm{C}$; FTIR (neat) $v_{\max }$ : 3406, 3075, 2979, 1750, 1593, 1330, 1204, $1085 \mathrm{~cm}^{-1} ;{ }^{1} \mathrm{H}$ NMR $\left(\mathrm{CDCl}_{3}, 400 \mathrm{MHz}\right): \delta=8.24(\mathrm{~d}, J=2.8 \mathrm{~Hz}, 1 \mathrm{H}, \mathrm{Ar})$, 8.09 (dd, $J=2.8,9.0 \mathrm{~Hz}, 1 \mathrm{H}, \mathrm{Ar}$ ), 7.20-7.11 (m, 2H, Ar), $6.76(\mathrm{~d}, J=9.0 \mathrm{~Hz}, 1 \mathrm{H}, \mathrm{Ar}), 6.71(\mathrm{t}, J=7.5 \mathrm{~Hz}, 1 \mathrm{H}, \mathrm{Ar})$, 6.67- $6.61(\mathrm{~m}, 2 \mathrm{H}, \mathrm{Ar}), 4.84$ (undivided $\mathrm{dd}, J=5.3$, $6.5 \mathrm{~Hz}, 1 \mathrm{H}, \mathrm{CH}), 4.50(\mathrm{~d}, J=15.9 \mathrm{~Hz}, 1 \mathrm{H}, \mathrm{CHH}, 1 / 2 \mathrm{H}$, $\mathrm{NH}: \mathrm{D}_{2} \mathrm{O}$ exchangeable), $4.41(\mathrm{~d}, J=15.9 \mathrm{~Hz}, 1 \mathrm{H}, \mathrm{CH} \underline{\mathrm{H}}$, $1 / 2 \mathrm{H}, \mathrm{NH}: \mathrm{D}_{2} \mathrm{O}$ exchangeable), $3.78\left(\mathrm{~s}, 3 \mathrm{H}, \mathrm{OCH}_{3}\right)$, 2.25-1.92 (m, 2H, $\left.\mathrm{CH}_{2}\right), 1.10\left(\mathrm{t}, J=7.4 \mathrm{~Hz}, 3 \mathrm{H}, \mathrm{CH}_{3}\right)$; ${ }^{13} \mathrm{C} \mathrm{NMR}\left(\mathrm{CDCl}_{3}, 101 \mathrm{MHz}\right): \delta=170.4(\mathrm{C}, \mathrm{C}-2), 160.0$ (C, C-5), 147.4 (C, C-13), 141.7 (C, C-8), 129.6 (C, C-10), 129.0 (C, C-15, C-17), 124.2 (C, C-7, C-9), 117.6 (C, C-16), 112.8 (C, C-14, C-18), 110.7 (C, C-6), $77.3(\mathrm{CH}$, $\mathrm{C}-3), 52.3\left(\mathrm{OCH}_{3}, \mathrm{C}-1\right), 42.8\left(\mathrm{CH}_{2}, \mathrm{C}-12\right), 25.7\left(\mathrm{CH}_{2} \mathrm{CH}_{3}\right.$, C-4), $9.2\left(\mathrm{CH}_{2} \mathrm{CH}_{3}, \mathrm{C}-4\right)$; GCMS m/z $344[\mathrm{M}]^{+}(90), 285$ (5), 243 (100), 227 (12), 197 (20), 180 (5), 167 (8), 152 (14), 134 (7), 106 (20) 93 (14), 77 (14), 59 (18); Anal. Calcd for $\mathrm{C}_{18} \mathrm{H}_{20} \mathrm{~N}_{2} \mathrm{O}_{5}: \mathrm{C}, 62.78 ; \mathrm{H}, 5.85 ; \mathrm{N}, 8.13$. Found: $\mathrm{C}, 62.65 ; \mathrm{H}, 5.87 ; \mathrm{N}, 8.26 . \mathbf{4 a} \cdot \mathrm{HCl}$ a white solid mp. $106-108{ }^{\circ} \mathrm{C}$.

Methyl 2-(4-nitro-2-((phenylamino)methyl)phenoxy)pentanoate (4b) Yellow solid $(\mathrm{MeOH})$ This compound $(\mathbf{4 b})$ was prepared from methyl 2-(4-nitro-2-((phenylimino)methyl)phenoxy)pentanoate (3b) $(0.56 \mathrm{mmol}, 0.20 \mathrm{~g})$ and sodium triacetoxyborohydride $(0.84 \mathrm{mmol}, 0.178 \mathrm{~g})$ according to the general procedure. The product obtained as a pale yellow solid was purified from methanol. $0.12 \mathrm{~g}$ (60\%); mp 74-76 ${ }^{\circ} \mathrm{C}$; FTIR (neat) $v_{\max }: 3408,3075,2979$, 1751，1593，1513，1328，1209, $1079 \mathrm{~cm}^{-1} ;{ }^{1} \mathrm{H}$ NMR $\left(\mathrm{CDCl}_{3}, 400 \mathrm{MHz}\right): \delta=8.25(\mathrm{~d}, J=2.7 \mathrm{~Hz}, 1 \mathrm{H}, \mathrm{Ar})$, $8.10(\mathrm{dd}, J=2.7,9.0 \mathrm{~Hz}, 1 \mathrm{H}, \mathrm{Ar}), 7.20-7.14(\mathrm{~m}, 2 \mathrm{H}, \mathrm{Ar})$, $6.76(\mathrm{~d}, J=9.1 \mathrm{~Hz}, 1 \mathrm{H}, \mathrm{Ar}), 6.72(\mathrm{t}, J=7.3 \mathrm{~Hz}, 1 \mathrm{H}, \mathrm{Ar})$, 6.67-6.62 (m, 2H, Ar), $4.872(\mathrm{dd}, J=7.2,5.0 \mathrm{~Hz}, 1 \mathrm{H}$, $\mathrm{CH}), \quad 4.50(\mathrm{~d}, \quad J=16.0 \mathrm{~Hz}, \quad 1 \mathrm{H}, \quad \mathrm{CHH}), \quad 4.41 \quad(\mathrm{~d}$, $J=16.0 \mathrm{~Hz}, 2 \mathrm{H} ; 1 \mathrm{H}, \mathrm{CHH}, 1 \mathrm{H} \mathrm{NH}: \mathrm{D}_{2} \mathrm{O}$ exchangeable), 3.77 (s, $\left.3 \mathrm{H}, \mathrm{OCH}_{3}\right), 2.11-1.94\left(\mathrm{~m}, 2 \mathrm{H}, \mathrm{CH}_{2}\right), 1.56$ (sext, $\left.J=7.5 \mathrm{~Hz}, 2 \mathrm{H}, \mathrm{CH}_{2}\right), 0.99\left(\mathrm{t}, J=7.4 \mathrm{~Hz}, 3 \mathrm{H}, \mathrm{CH}_{3}\right) ;{ }^{13} \mathrm{C}$ NMR $\left(\mathrm{CDCl}_{3}, 101 \mathrm{MHz}\right): \delta=171.0(\mathrm{C}, \mathrm{C}-2), 160.3(\mathrm{C}$, C-5), 147.7 (C, C-13), 142.00 (C, C-8), 129.9 (C, C-10),
129.3 (C, C-15, C-17), 124.5 (C, C-7, C-9), 117.9 (C, C-16), 113.1 (C, C-14, C-18), 110.9 (C, C-6), $76.5(\mathrm{CH}$, $\mathrm{C}-3), 52.2\left(\mathrm{OCH}_{3}, \mathrm{C}-1\right), 43.1\left(\mathrm{CH}_{2}, \mathrm{C}-12\right), 34.5\left(\mathrm{CH}_{2-}\right.$ $\left.\mathrm{CH}_{2} \mathrm{CH}_{3}, \mathrm{C}-4\right), 18.5\left(\mathrm{CH}_{2} \mathrm{CH}_{2} \mathrm{CH}_{3}, \mathrm{C}-4\right), 13.7\left(\mathrm{CH}_{2} \mathrm{CH}_{2-}\right.$ $\left.\mathrm{CH}_{3}, \mathrm{C}-4\right)$; GCMS m/z 358 [M] ${ }^{+}$(93), 329 (8), 299 (6), 243 (100), 227 (11), 197 (19), 167 (7), 152 (10), 106 (15), 93 (14), 77 (9), 55 (9); FTIR $\left(\mathrm{cm}^{-1}\right)$ : 3408, 3110-3018, 2958-2871, 1751, 1593, 1513, 1328, 1209, 1079-1059; Anal. Calcd for $\mathrm{C}_{19} \mathrm{H}_{22} \mathrm{~N}_{2} \mathrm{O}_{5}$ : C, 66.04; H, 5.85; N, 8.56. Found: $\mathrm{C}, 66.80 ; \mathrm{H}, 5.72 ; \mathrm{N}, 8.80 . \mathbf{4 b} \cdot \mathrm{HCl}$ a white solid mp. $106-108{ }^{\circ} \mathrm{C}$.

Methyl 2-(4-nitro-2-((phenylamino)methyl)phenoxy)hexanoate (4c) Yellow solid $(\mathrm{MeOH})$ This compound (4c) was prepared from methyl 2-(4-nitro-2-((phenylimino)methyl)phenoxy)hexanoate (3c) $(0.54 \mathrm{mmol}, 0.20 \mathrm{~g})$ and sodium triacetoxyborohydride $(0.81 \mathrm{mmol}, 0.171 \mathrm{~g})$ according to the general procedure. The product obtained as a pale yellow solid was purified from methanol. $0.11 \mathrm{~g}$ (55\%); mp 42-44 ${ }^{\circ} \mathrm{C}$; FTIR (neat) $v_{\max }: 3416,3051,2955$, 1742, 1592, 1512, 1337, 1203, $1082 \mathrm{~cm}^{-1}$; ${ }^{1} \mathrm{H}$ NMR $\left(\mathrm{CDCl}_{3}, 400 \mathrm{MHz}\right): \delta=8.24(\mathrm{~d}, J=2.7 \mathrm{~Hz}, 1 \mathrm{H}, \mathrm{Ar})$, 8.09 (dd, $J=2.7,9.0 \mathrm{~Hz}, 1 \mathrm{H}, \mathrm{Ar}), 7.20-7.12(\mathrm{~m}, 2 \mathrm{H}, \mathrm{Ar})$, $6.76(\mathrm{~d}, J=9.0 \mathrm{~Hz}, 1 \mathrm{H}, \mathrm{Ar}), 6.71(\mathrm{t}, J=7.3 \mathrm{~Hz}, 1 \mathrm{H}, \mathrm{Ar})$, $6.64(\mathrm{~d}, J=8.5 \mathrm{~Hz}, 2 \mathrm{H}, \mathrm{Ar}), 4.87(\mathrm{t}, J=6.0 \mathrm{~Hz}, 1 \mathrm{H}, \mathrm{CH})$, $4.49(\mathrm{~d}, J=15.9 \mathrm{~Hz}, 1 \mathrm{H}, \mathrm{CHH}), 4.41(\mathrm{~d}, J=15.9 \mathrm{~Hz}$, $1 \mathrm{H}, \mathrm{CHH}), 3.77\left(\mathrm{~s}, 3 \mathrm{H}, \mathrm{OCH}_{3}\right), 2.08-1.98\left(\mathrm{~m}, 2 \mathrm{H}, \mathrm{CH}_{2}\right)$, $1.57-1 . \overline{45}\left(\mathrm{~m}, 2 \mathrm{H}, \mathrm{CH}_{2}\right), 1.45-1.32\left(\mathrm{~m}, 2 \mathrm{H}, \mathrm{CH}_{2}\right), 0.92(\mathrm{t}$, $\left.J=7.2 \mathrm{~Hz}, 3 \mathrm{H}, \mathrm{CH}_{3}\right) ;{ }^{13} \mathrm{C} \mathrm{NMR}\left(\mathrm{CDCl}_{3}, 101 \mathrm{MHz}\right)$ : $\delta=171.0(\mathrm{C}, \mathrm{C}-2), 160.4$ (C, C-5), 147.7 (C, C-13), 142.0 (C, C-8), 129.9 (C, C-10), 129.3 (C, C-15, C-17), 124.6 (C, C-9), 124.5 (C, C-7), 117.9 (C, C-16), 113.1 (C, C-14, $\mathrm{C}-18), 111.0$ (C, C-6), $76.7(\mathrm{CH}, \mathrm{C}-3), 52.6\left(\mathrm{OCH}_{3}, \mathrm{C}-1\right)$, $43.2\left(\mathrm{CH}_{2}, \mathrm{C}-12\right), 32.3\left(\mathrm{CH}_{2} \mathrm{CH}_{2} \mathrm{CH}_{2} \mathrm{CH}_{3}, \mathrm{C}-4\right), 27.3$ $\left(\mathrm{CH}_{2} \mathrm{CH}_{2} \mathrm{CH}_{2} \mathrm{CH}_{3}, \mathrm{C}-4\right), 22.3 \quad\left(\mathrm{CH}_{2} \mathrm{CH}_{2} \mathrm{CH}_{2} \mathrm{CH}_{3}, \mathrm{C}-4\right)$, $13.8\left(\mathrm{CH}_{2} \mathrm{CH}_{2} \mathrm{CH}_{2} \mathrm{CH}_{3}, \mathrm{C}-4\right)$; GCMS $m / z 372[\mathrm{M}]^{+}(90)$, 329 (8), 243 (100), 227 (11), 197 (17), 167 (6), 152 (8), 106 (13), 93 (15), 69 (10), 59 (6); Anal. Calcd for $\mathrm{C}_{20} \mathrm{H}_{24} \mathrm{~N}_{2} \mathrm{O}_{5}$ : C, 64.50; H, 6.50; N, 7.52. Found: C, 64.455; H, 6.62; N, 7.55. $4 \mathbf{c} \cdot \mathrm{HCl}$ a white solid mp. $106-108{ }^{\circ} \mathrm{C}$.

Methyl 2-(2-methoxy-4-nitro-6-((phenylamino)methyl)phenoxy)butanoate (4d) Yellow solid ( $\mathrm{MeOH})$ This compound (4d) was prepared from methyl 2-(2-methoxy4-nitro-6-((phenylimino)methyl)phenoxy)butanoate (3d) $(0.54 \mathrm{mmol}, 0.20 \mathrm{~g})$ and sodium triacetoxyborohydride $(0.81 \mathrm{mmol}, 0.171 \mathrm{~g})$ according to the general procedure. The product obtained as a pale yellow solid was purified from methanol. $0.11 \mathrm{~g} \mathrm{(55 \% );} \mathrm{mp} 117-119^{\circ} \mathrm{C}$; FTIR (neat) $v_{\max }: 3387,3051,2955,1743,1601,1514,1332$, 1204, $1091 \mathrm{~cm}^{-1}$; ${ }^{1} \mathrm{H}$ NMR $\left(\mathrm{CDCl}_{3}, 400 \mathrm{MHz}\right): \delta=7.93$ $(\mathrm{d}, J=2.6 \mathrm{~Hz}, 1 \mathrm{H}, \mathrm{Ar}), 7.69(\mathrm{~d}, J=2.6 \mathrm{~Hz}, 1 \mathrm{H}, \mathrm{Ar})$, 7.20-7.11 (m, 2H, Ar), 6.74-6.68 (m, 1H, Ar), 6.67-6.60 (m, 2H, Ar), 5.26 (undivided dd, $J=5.6,6.8 \mathrm{~Hz}, 1 \mathrm{H}, \mathrm{CH}$ ), 
$4.57(\mathrm{~d}, J=16.0 \mathrm{~Hz}, 1 \mathrm{H}, \mathrm{CHH}), 4.48(\mathrm{~d}, J=16.0 \mathrm{~Hz}$, $1 \mathrm{H}, \mathrm{CHH}), 4.38$ (s, $1 \mathrm{H}, \mathrm{NH}: \overline{\mathrm{D}}_{2} \mathrm{O}$ exchangeable), $3.89(\mathrm{~s}$, $\left.3 \mathrm{H}, \mathrm{OC}_{3}\right), 3.73\left(\mathrm{~s}, 3 \mathrm{H}, \mathrm{OCH}_{3}\right), 2.10-1.92\left(\mathrm{~m}, 2 \mathrm{H}, \mathrm{CH}_{2}\right)$, $1.08\left(\mathrm{t}, J=7.4 \mathrm{~Hz}, 3 \mathrm{H}, \mathrm{CH}_{3}\right) ;{ }^{13} \mathrm{C} \quad \mathrm{NMR}\left(\mathrm{CDCl}_{3}\right.$, $101 \mathrm{MHz}): \delta=171.8$ (C, C-2), 150.5 (C, C-6), 149.8 (C, C-5), 147.8 (C, C-13), 143.0 (C, C-8), 133.7 (C, C-10), 129.3 (C, C-15, C-17), 117.8 (C, C-16), 116.8 (C, C-9), 113.0 (C, C-14, C-18), 107.0 (C, C-7), 80.6 (CH, C-3), 56.2 $\left(\mathrm{OCH}_{3}, \mathrm{C}-11\right), 52.1\left(\mathrm{OCH}_{3}, \mathrm{C}-1\right), 43.4\left(\mathrm{CH}_{2}, \mathrm{C}-12\right), 26.7$ $\left(\mathrm{CH}_{2} \mathrm{CH}_{3}, \mathrm{C}-4\right), 9.3\left(\mathrm{CH}_{2} \underline{\mathrm{CH}}_{3}, \mathrm{C}-4\right)$; GCMS $\mathrm{m} / z, 374[\mathrm{M}]^{+}$ (62), 273 (100), 257 (37), 227 (16), 207 (7), 182 (12), 154 (7), 134 (7), 106 (17), 93 (26), 77 (12), 59 (14); Anal. Calcd for $\mathrm{C}_{19} \mathrm{H}_{22} \mathrm{~N}_{2} \mathrm{O}_{6}: \mathrm{C}, 60.95 ; \mathrm{H}, 5.92 ; \mathrm{N}, 7.48$. Found: C, 61.05; H, 5.99; N, 7.42. $4 \mathbf{d} \cdot \mathrm{HCl}$ a white solid mp. $106-108{ }^{\circ} \mathrm{C}$.

Methyl 2-(2-methoxy-4-nitro-6-((phenylamino)methyl)phenoxy)pentanoate $(\mathbf{4 e})$ Yellow solid $(\mathrm{MeOH})$ This compound (4e) was prepared from methyl 2-(2-methoxy-4nitro-6-((phenylimino)methyl)phenoxy)pentanoate (3e) $(0.52 \mathrm{mmol}, 0.20 \mathrm{~g})$ and sodium triacetoxyborohydride $(0.78 \mathrm{mmol}, 0.166 \mathrm{~g})$ according to the general procedure. The product obtained as a pale yellow solid was purified from methanol. $0.11 \mathrm{~g}(55 \%), \mathrm{mp} 112-114{ }^{\circ} \mathrm{C}$; FTIR (neat) $v_{\max }: 3397,3051,2955,1746,1602,1514,1336$, 1203, $1097 \mathrm{~cm}^{-1} ;{ }^{1} \mathrm{H} \mathrm{NMR}\left(\mathrm{CDCl}_{3}, 400 \mathrm{MHz}\right): \delta=7.93$ (d, $J=2.5 \mathrm{~Hz}, 1 \mathrm{H}, \mathrm{Ar}), 7.68(\mathrm{~d}, J=2.5 \mathrm{~Hz}, 1 \mathrm{H}, \mathrm{Ar})$, 7.20-7.13 (m, 2H, Ar), 6.76-6.58 (m, 1H, Ar), 6.67-6.58 $(\mathrm{m}, 2 \mathrm{H}, \mathrm{Ar}), 5.31(\mathrm{t}, J=6.0 \mathrm{~Hz}, 1 \mathrm{H}, \mathrm{CH}), 4.59(\mathrm{~d}$, $J=15.9 \mathrm{~Hz}, 1 \mathrm{H}, \mathrm{CHH}), 4.55(\mathrm{~d}, J=15.9 \mathrm{~Hz}, 1 \mathrm{H}, \mathrm{CHH})$, 4.37 (bs, $1 \mathrm{H}, \mathrm{NH}: \mathrm{D}_{2} \mathrm{O}$ exchangeable), 3.89 (s, $3 \mathrm{H}, \mathrm{OCH}_{3}$ ), $3.72\left(\mathrm{~s}, 3 \mathrm{H}, \mathrm{OCH}_{3}\right), 2.03-1.88\left(\mathrm{~m}, 2 \mathrm{H}, \mathrm{CH}_{2}\right), 1.66-1.43$ $\left(\mathrm{m}, 2 \mathrm{H}, \mathrm{CH}_{2}\right), 0.97\left(\mathrm{t}, J=7.4 \mathrm{~Hz}, 3 \mathrm{H}, \mathrm{CH}_{3}\right) ;{ }^{13} \mathrm{C} \mathrm{NMR}$ $\left(\mathrm{CDCl}_{3}, 101 \mathrm{MHz}\right): \delta=172.0(\mathrm{C}, \mathrm{C}-2), 150.5(\mathrm{C}, \mathrm{C}-6)$, 149.7 (C, C-5), 147.8 (C, C-13), 143.0 (C, C-8), 133.7 (C, C-10), 129.3 (C, C-15, C-17), 117.8 (C, C-16), 116.8 (C, C-9), 113.0 (C, C-14, C-18), 107.0 (C, C-7), 79.4 (CH, $\mathrm{C}-3), 56.2\left(\mathrm{OCH}_{3}, \mathrm{C}-11\right), 52.1\left(\mathrm{OCH}_{3}, \mathrm{C}-1\right), 43.4\left(\mathrm{CH}_{2}\right.$, $\mathrm{C}-12), 35.4\left(\mathrm{CH}_{2} \mathrm{CH}_{2} \mathrm{CH}_{3}, \mathrm{C}-4\right), 18.3\left(\mathrm{CH}_{2} \mathrm{CH}_{2} \mathrm{CH}_{3}, \mathrm{C}-4\right)$, $13.8\left(\mathrm{CH}_{2} \mathrm{CH}_{2} \mathrm{CH}_{3}, \mathrm{C}-4\right)$; GCMS $388 \mathrm{~m} / z$ [M] ${ }^{+}$(52), 273 (100), 257 (33), 227 (14), 207 (9), 182 (10), 148 (6), 106 (15), 93 (29), 87 (5), 77 (11), 73 (7), 59 (8), 55 (12); Anal. Calcd for $\mathrm{C}_{20} \mathrm{H}_{24} \mathrm{~N}_{2} \mathrm{O}_{6}$ : C, 61.84; $\mathrm{H}, 6.23 ; \mathrm{N}, 7.21$. Found: $\mathrm{C}, 61.65 ; \mathrm{H}, 6.28 ; \mathrm{N}, 7.15 .4 \mathbf{e} \cdot \mathrm{HCl}$ a white solid mp. $106-108{ }^{\circ} \mathrm{C}$.

Methyl 2-(2-methoxy-4-nitro-6-((phenylamino)methyl)phenoxy)hexanoate $(\mathbf{4 f})$ Yellow solid $(\mathrm{MeOH})$ This compound (4f) was prepared from methyl 2-(2-methoxy-4nitro-6-((phenylimino)methyl)phenoxy)hexanoate (3f) $(0.50 \mathrm{mmol}, 0.20 \mathrm{~g})$ and sodium triacetoxyborohydride $(0.75 \mathrm{mmol}, 0.16 \mathrm{~g})$ according to the general procedure. The product obtained as a pale yellow solid was purified from methanol. $0.15 \mathrm{~g}$ (85\%); mp $119-121{ }^{\circ} \mathrm{C}$; FTIR (neat) $v_{\max }: 3385,3091,2953,1746,1601,1514,1334$, $1202,1095 \mathrm{~cm}^{-1} ;{ }^{1} \mathrm{H}$ NMR $\left(\mathrm{CDCl}_{3}, 400 \mathrm{MHz}\right): \delta=7.92$ $(\mathrm{d}, J=2.6 \mathrm{~Hz}, 1 \mathrm{H}, \mathrm{Ar}), 7.68(\mathrm{~d}, J=2.6 \mathrm{~Hz}, 1 \mathrm{H}, \mathrm{Ar})$, 7.20-7.11 (m, 2H, Ar), 6.70 (t, $J=7.3 \mathrm{~Hz}, 1 \mathrm{H}, \mathrm{Ar}), 6.63$ (d, $J=7.8 \mathrm{~Hz}, 2 \mathrm{H}, \mathrm{Ar}$ ), 5.29 (undivided $\mathrm{dd}, J=5.6$, $6.8 \mathrm{~Hz}, 1 \mathrm{H}, \mathrm{CH}), 4.56(\mathrm{~d}, J=15.8 \mathrm{~Hz}, 1 \mathrm{H}, \mathrm{CHH}), 4.47$ (d, $J=15.9 \mathrm{~Hz}, \quad 1 \mathrm{H}, \quad \mathrm{CHH}), 4.36 \quad\left(\mathrm{bs}, \quad 1 \mathrm{H}, \quad \mathrm{NH}: \quad \mathrm{D}_{2} \mathrm{O}\right.$ exchangeable), $3.88\left(\mathrm{~s}, \overline{3} \mathrm{H}, \mathrm{OCH}_{3}\right), 3.72\left(\mathrm{~s}, 3 \mathrm{H}, \mathrm{OCH}_{3}\right)$, 2.02-1.89 (m, 2H, $\left.\mathrm{CH}_{2}\right), 1.55-1.30\left(\mathrm{~m}, 4 \mathrm{H}, \mathrm{C}_{2} \mathrm{H}_{4}\right), 0.90(\mathrm{t}$, $\left.J=7.2 \mathrm{~Hz}, 3 \mathrm{H}, \mathrm{CH}_{3}\right) ;{ }^{13} \mathrm{C} \mathrm{NMR}\left(\mathrm{CDCl}_{3}, 101 \mathrm{MHz}\right)$ : $\delta=172.0(\mathrm{C}, \mathrm{C}-2), 150.5$ (C, C-6), 149.8 (C, C-5), 147.8 (C, C-13), 143.0 (C, C-8), 133.7 (C, C-10), 129.3 (C, C-15, C-17), 117.8 (C, C-16), 116.8 (C, C-9), 113.0 (C, C-14, $\mathrm{C}-18), 107.0$ (C, C-7), 79.7 (CH, C-3), $56.2\left(\mathrm{OCH}_{3}, \mathrm{C}-11\right)$, $52.1\left(\mathrm{OCH}_{3}, \mathrm{C}-1\right), 43.4\left(\mathrm{CH}_{2}, \mathrm{C}-12\right), 33.1\left(\mathrm{CH}_{2} \mathrm{CH}_{2} \mathrm{CH}_{2}\right.$ $\left.\mathrm{CH}_{3}, \mathrm{C}-4\right), 27.0\left(\mathrm{CH}_{2} \mathrm{CH}_{2} \mathrm{CH}_{2} \mathrm{CH}_{3}, \mathrm{C}-4\right), 22.4\left(\mathrm{CH}_{2} \mathrm{CH}_{2}\right.$ $\left.\mathrm{CH}_{2} \mathrm{CH}_{3}, \mathrm{C}-4\right)$, $13.9 \quad\left(\mathrm{CH}_{2} \mathrm{CH}_{2} \mathrm{CH}_{2} \mathrm{CH}_{3}, \mathrm{C}-4\right) ; \mathrm{GCMS}$ $402 \mathrm{~m} / z\left[^{[\mathrm{M}]^{+}}\right.$(46), 273 (100), 257 (32), 227 (13), 182 (9), 154 (5), 106 (12), 93 (25), 69 (9), 55 (6); Anal. Calcd for $\mathrm{C}_{21} \mathrm{H}_{26} \mathrm{~N}_{2} \mathrm{O}_{6}$ (402.18): C, 62.67; H, 6.51; N, 6.96. Found: $\mathrm{C}, 62.72 ; \mathrm{H}, 6.45 ; \mathrm{N}, 6.90 .4 \mathbf{4 f} \cdot \mathrm{HCl}$ a white solid mp. $106-108{ }^{\circ} \mathrm{C}$.

\section{Direct reductive amination of 1a-f; synthesis of (4a-f)}

Aniline $(1.87 \mathrm{mmol})$, sodium triacetoxyborohydride $(2.81 \mathrm{mmol})$, and acetic acid $(0.2 \mathrm{~mL})$ were added to a mixture of methyl 2-(2-formyl-4-nitrophenoxy)alkanoate (1a-f) $(1.87 \mathrm{mmol})$ in 1,2-dichloroethane $(15 \mathrm{~mL})$. The mixture was stirred magnetically at room temperature for $4 \mathrm{~h}$. Next, the mixture was washed (neutralized) with an aqueous solution of sodium carbonate $5 \%\left(\mathrm{NaHCO}_{3}\right)$ $(30 \mathrm{~mL})$ and extracted with ethyl acetate. The resultant organic layer was dried and evaporated, and residue was recrystallized from methanol to give $\mathbf{4 a}-\mathbf{f}$. Yields of $\mathbf{4 a}-\mathbf{f}$ are given in Scheme 4.

\section{General procedure for the synthesis of methyl 2-(4- amino-2-((phenylamino)methyl)phenoxy)alkanoate (5a and $5 \mathrm{c}-\mathrm{f})$}

A mixture of methanol $(10 \mathrm{~mL})$ and $10 \% \mathrm{Pd} / \mathrm{C}$ catalyst $(0.033 \mathrm{~g})$ was stirred magnetically under a slow stream of hydrogen ( 1 bubble per second) at room temperature for $30 \mathrm{~min}$. Then, a solution of $\mathbf{3 a}$ or $\mathbf{3 c}-\mathbf{f}(0.91 \mathrm{mmol})$ in methanol $(10 \mathrm{~mL})$ and DME $(10 \mathrm{~mL})$ was added, and reduction was carried out until the conversion of $\mathbf{3 a}$ or $\mathbf{3} \mathbf{c}-$ f was completed $(7 \mathrm{~h})$, which was determined by gas chromatography. The mixture was left overnight, the catalyst was separated on the next day, the solvent was evaporated, and the residue was obtained to give 5a and 5c-f. 
Methyl 2-(4-amino-2-((phenylamino)methyl)phenoxy)butanoate (5a) Brown semi-solid This compound (5a) was prepared from methyl 2-(4-nitro-2-((phenylimino)methyl)phenoxy)butanoate (3a) (0.91 mmol, $0.31 \mathrm{~g})$ and $10 \% \mathrm{Pd} / \mathrm{C}$ catalyst $(0.031 \mathrm{~g})$ according to the general procedure. The product obtained as a brown semi-solid $0243 \mathrm{~g}(85 \%)$; FTIR (neat) $v_{\max }: 3365,3019,2935,1736$, 1599, 1202, $1058 \mathrm{~cm}^{-1}$; ${ }^{1} \mathrm{H}$ NMR $\left(\mathrm{CDCl}_{3}, 400 \mathrm{MHz}\right)$ : $\delta=7.18-7.09(\mathrm{~m}, 2 \mathrm{H}, \mathrm{Ar}), 6.72-6.54(\mathrm{~m}, 5 \mathrm{H}, \mathrm{Ar}), 6.46$ $(\mathrm{dd}, J=2.7,8.5 \mathrm{~Hz}, 1 \mathrm{H}, \mathrm{Ar}), 4.59(\mathrm{t}, J=6.0 \mathrm{~Hz}, 1 \mathrm{H}$, $\mathrm{CH}), \quad 4.36(\mathrm{~d}, \quad J=14.6 \mathrm{~Hz}, 1 \mathrm{H}, \mathrm{CHH}), 4.26 \quad(\mathrm{~d}$, $J=14.6 \mathrm{~Hz}, 1 \mathrm{H}, \mathrm{CH} \underline{\mathrm{H}}), 3.90-3.53\left(\mathrm{~m}, 5 \overline{\mathrm{H}} ; 3 \mathrm{H}, \mathrm{OCH}_{3}, 2 \mathrm{H}\right.$ $\mathrm{NH}_{2}$ : $\mathrm{D}_{2} \mathrm{O}$ exchangeable), 2.01-1.91 (m, 2H, $\left.\mathrm{CH}_{2}\right), 1.04(\mathrm{t}$, $\left.J=7.4 \mathrm{~Hz}, 3 \mathrm{H}, \mathrm{CH}_{3}\right) ;{ }^{13} \mathrm{C} \mathrm{NMR}\left(\mathrm{CDCl}_{3}, 101 \mathrm{MHz}\right)$ : $\delta=172.5$ (C, C-2), 148.6 (C, C-5), 148.5 (C, C-13), 140.7 (C, C-8), 129.4 (C, C-10), 129.1 (C, C-15, C-17), 117.2 (C, C-16), 116.7 (C, C-9), 114.2 (C, C-7), 113.2 (C, C-6), 113.1 (C, C-14, C-18), $77.9(\mathrm{CH}, \mathrm{C}-3), 52.1\left(\mathrm{OCH}_{3}, \mathrm{C}-1\right)$, $43.7\left(\mathrm{CH}_{2}, \mathrm{C}-12\right), 26.3\left(\mathrm{CH}_{2} \mathrm{CH}_{3}, \mathrm{C}-4\right), 9.6\left(\mathrm{CH}_{2} \mathrm{CH}_{3}\right.$, C-4); GCMS $314 \mathrm{~m} / z[\mathrm{M}]^{+}$(100), 213 (46), 196 (30), 162 (36) 134 (16), 122 (59), 106 (11), 104 (25), 93 (24), 77 (18), 59 (9); Anal. Calcd for $\mathrm{C}_{18} \mathrm{H}_{22} \mathrm{~N}_{2} \mathrm{O}_{3}$ : C, 68.77; $\mathrm{H}$, 7.05; N, 8.91. Found: C, 68.64; H, 7.15; N, 8.95.

Methyl 2-(4-amino-2-((phenylamino)methyl)phenoxy)hexanoate (5c) Brown semi-solid This compound (5c) was prepared from methyl 2-(4-nitro-2-((phenylimino)methyl)phenoxy)hexanoate (3c) $(0.54 \mathrm{mmol}, 0.20 \mathrm{~g})$ and $10 \% \mathrm{Pd} / \mathrm{C}$ catalyst $(0.02 \mathrm{~g})$ according to the general procedure. The product obtained as a brown semi-solid $0.151 \mathrm{~g}(82 \%)$; FTIR (neat) $v_{\max }: 3365,3019,2935,1738$, 1600, 1201, $1063 \mathrm{~cm}^{-1}$; ${ }^{1} \mathrm{H}$ NMR $\left(\mathrm{CDCl}_{3}, 400 \mathrm{MHz}\right)$ : $\delta=7.19-7.10(\mathrm{~m}, 2 \mathrm{H}, \mathrm{Ar}), 6.72-6.54$ (m, 5H, Ar), 6.46 (dd, $J=2.8,8.5 \mathrm{~Hz}, 1 \mathrm{H}, \mathrm{Ar}$ ), 4.63 (undivided dd, $J=5.6$, $6.8 \mathrm{~Hz}, 1 \mathrm{H}, \mathrm{CH}), 4.35$ (d, $J=14.5 \mathrm{~Hz}, 1 \mathrm{H}, \mathrm{CHH}), 4.26(\mathrm{~d}$, $J=14.5 \mathrm{~Hz}, 1 \mathrm{H}, \mathrm{CHH}), 3.71\left(\mathrm{~s}, 4 \mathrm{H} ; 3 \mathrm{H}, \mathrm{OCH}_{3}, 1 \mathrm{H} \mathrm{NH}\right.$ : $\mathrm{D}_{2} \mathrm{O}$ exchangeable $), 1.98-1.87\left(\mathrm{~m}, 2 \mathrm{H}, \mathrm{CH}_{2}\right), 1.51-1.41$ $\left(\mathrm{m}, 2 \mathrm{H}, \mathrm{CH}_{2}\right), \quad 1.40-1.27\left(\mathrm{~m}, 2 \mathrm{H}, \mathrm{CH}_{2}\right), 0.88(\mathrm{t}$, $\left.J=7.2 \mathrm{~Hz}, 3 \mathrm{H}, \mathrm{CH}_{3}\right) ;{ }^{13} \mathrm{C} \mathrm{NMR}\left(\mathrm{CDCl}_{3}, 101 \mathrm{MHz}\right)$ : $\delta=172.8$ (C, C-2), 148.6 (C, C-5), 148.6 (C, C-13), 140.7 (C, C-8), 129.4 (C, C-10), 129.1 (C, C-15, C-17), 117.2 (C, C-16), 116.7 (C, C-9), 114.2 (C, C-7), 113.2 (C, C-6), 113.1 (C, C-14, C-18), 76.9 (CH, C-3), $52.1\left(\mathrm{OCH}_{3}, \mathrm{C}-1\right)$, $43.7\left(\mathrm{CH}_{2}, \mathrm{C}-12\right), 32.7\left(\mathrm{CH}_{2} \mathrm{CH}_{2} \mathrm{CH}_{2} \mathrm{CH}_{3}, \mathrm{C}-4\right), 27.4$ $\left(\mathrm{CH}_{2} \mathrm{CH}_{2} \mathrm{CH}_{2} \mathrm{CH}_{3}, \mathrm{C}-4\right), 22.4\left(\mathrm{CH}_{2} \mathrm{CH}_{2} \mathrm{CH}_{2} \mathrm{CH}_{3}, \mathrm{C}-4\right)$, $13.9\left(\mathrm{CH}_{2} \mathrm{CH}_{2} \mathrm{CH}_{2} \mathrm{CH}_{3}, \mathrm{C}-4\right)$; GCMS $342 \mathrm{~m} / \mathrm{z}[\mathrm{M}]^{+}$(100), 213 (63), 196 (35), 162 (9), 134 (7), 122 (49), 106 (11), 104 (26), 93 (27), 77 (17), 55 (7); Anal. Calcd for $\mathrm{C}_{20} \mathrm{H}_{26} \mathrm{~N}_{2} \mathrm{O}_{3}$ : C, 70.15; H, 7.65; N, 8.18. Found: C, 70.35; H, 7.72; N, 8.16 .

Methyl 2-(4-amino-2-methoxy-6-((phenylamino)methyl)phenoxy)butanoate (5d) Brown semi-solid This compound (5d) was prepared from methyl 2-(2-methoxy-4- nitro-6-((phenylimino)methyl)phenoxy)butanoate

(3d) $(0.54 \mathrm{mmol}, 0.20 \mathrm{~g})$ and $10 \% \mathrm{Pd} / \mathrm{C}$ catalyst $(0.02 \mathrm{~g})$ according to the general procedure. The product obtained as a brown semi-solid $0.132 \mathrm{~g}(71 \%)$; FTIR (neat) $v_{\max }$ : 3365, 3019, 2935, 1737, 1599, 1199, $1054 \mathrm{~cm}^{-1} ;{ }^{1} \mathrm{H}$ NMR $\left(\mathrm{CDCl}_{3}, 400 \mathrm{MHz}\right): \delta=7.28-6.99(\mathrm{~m}, 2 \mathrm{H}, \mathrm{Ar}) ; 6.81-6.57$ $(\mathrm{m}, 3 \mathrm{H}, \mathrm{Ar}), 6.24(\mathrm{~d}, J=2.5 \mathrm{~Hz}, 1 \mathrm{H}, \mathrm{Ar}), 6.13(\mathrm{~d}$, $J=2.5 \mathrm{~Hz}, 1 \mathrm{H}, \mathrm{Ar}), 4.72-4.66(\mathrm{~m}, 1 \mathrm{H}, \mathrm{CH}), 4.51-4.29$ (m, 2H, $\mathrm{CH}_{2}$ ), 3.76-3.54 (m, 8H; $6 \mathrm{H} \mathrm{OCH}_{3}, 2 \mathrm{H} \mathrm{NH} \mathrm{NH}_{2} \mathrm{O}$ exchangeable), 2.03-1.83 (m, 2H, $\left.\mathrm{CH}_{2}\right), 1.08-0.83(\mathrm{~m}, 4 \mathrm{H}$, $\left.3 \mathrm{H} \quad \mathrm{CH}_{3}, 1 \mathrm{H} \quad \mathrm{NH}\right) ;{ }^{13} \mathrm{C} \quad \mathrm{NMR} \quad\left(\mathrm{CDCl}_{3}, 101 \mathrm{MHz}\right)$ : $\delta=172.8$ (C, C-2), 152.1 (C, C-6), 148.5 (C, C-13), 142.8 (C, C-8), 137.3 (C, C-5), 133.5 (C, C-10), 129.1 (C, C-15, C-17), 117.1 (C, C-16), 112.9 (C, C-14, C-18), 106.8 (C, C-9), 99.2 (C, C-7), $81.6(\mathrm{CH}, \mathrm{C}-3), 55.6\left(\mathrm{OCH}_{3}, \mathrm{C}-11\right)$, $51.7\left(\mathrm{OCH}_{3}, \mathrm{C}-1\right), 43.4\left(\mathrm{CH}_{2}, \mathrm{C}-12\right), 26.5\left(\mathrm{CH}_{2} \mathrm{CH}_{3}, \mathrm{C}-4\right)$, $9.2\left(\mathrm{CH}_{2} \mathrm{CH}_{3}, \mathrm{C}-4\right)$; Anal. Calcd for $\mathrm{C}_{19} \mathrm{H}_{24} \mathrm{~N}_{2} \mathrm{O}_{4}$ : C, 66.26; H, 7.02; N, 8.13. Found: C, 66.36; H, 7.14; N, 8.23.

Methyl 2-(4-amino-2-methoxy-6-((phenylamino)methyl) phenoxy)pentanoate (5e) Brown semi-solid This compound (5e) was prepared from methyl 2-(2-methoxy4-nitro-6-((phenylimino)methyl)phenoxy)pentanoate (3e) $(0.93 \mathrm{mmol}, 0.36 \mathrm{~g})$ and $10 \% \mathrm{Pd} / \mathrm{C}$ catalyst $(0.036 \mathrm{~g})$ according to the general procedure. The product obtained as a brown semi-solid $0.28 \mathrm{~g}(84 \%)$; FTIR (neat) $v_{\max }$ : 3370, 3048, 2956, 1739, 1599, 1198, $1057 \mathrm{~cm}^{-1} ;{ }^{1} \mathrm{H}$ NMR $\left(\mathrm{CDCl}_{3}, 400 \mathrm{MHz}\right): \delta=7.27-7.02(\mathrm{~m}, 2 \mathrm{H}, \mathrm{Ar}), 6.71-6.57$ $(\mathrm{m}, 3 \mathrm{H}, \mathrm{Ar}), 6.24(\mathrm{~d}, J=2.5 \mathrm{~Hz}, 1 \mathrm{H}, \mathrm{Ar}), 6.12$ (d, $J=2.5 \mathrm{~Hz}, 1 \mathrm{H}, \mathrm{Ar}), 4.74(\mathrm{t}, J=6.2 \mathrm{~Hz}, 1 \mathrm{H}, \mathrm{CH})$, 4.43-4.32 (m, 2H, $\left.\mathrm{CH}_{2}\right), 3.78-3.63\left(\mathrm{~m}, 8 \mathrm{H} ; 6 \mathrm{H}, \mathrm{OCH}_{3}, 2 \mathrm{H}\right.$, $\mathrm{NH}_{2}: \quad \mathrm{D}_{2} \mathrm{O}$ exchangeable), 2.10-1.73 (m, 2H, $\left.\mathrm{CH}_{2}\right)$, 1.73-1.35 (m, 2H, $\left.\mathrm{CH}_{2}\right), 1.06-0.78\left(\mathrm{~m}, 4 \mathrm{H} ; 3 \mathrm{H}, \mathrm{CH}_{3}, 1 \mathrm{H}\right.$, $\mathrm{NH}: \mathrm{D}_{2} \mathrm{O}$ exchangeable $) ;{ }^{13} \mathrm{C} \mathrm{NMR}\left(\mathrm{CDCl}_{3}, 101 \mathrm{MHz}\right)$ : $\delta=173.0$ (C, C-2), 152.1 (C, C-6), 148.5 (C, C-13), 142.8 (C, C-8), 137.2 (C, C-5), 133.5 (C, C-10), 129.1 (C, C-15, C-17), 117.1 (C, C-16), 112.9 (C, C-14, C-18), 106.8 (C, C-9), 99.2 (C, C-7), $80.3(\mathrm{CH}, \mathrm{C}-3), 55.6\left(\mathrm{OCH}_{3}, \mathrm{C}-11\right)$, $51.7\left(\mathrm{OCH}_{3}, \mathrm{C}-1\right), 43.4\left(\mathrm{CH}_{2}, \mathrm{C}-12\right), 35.4\left(\mathrm{CH}_{2} \mathrm{CH}_{2} \mathrm{CH}_{3}\right.$, C-4), $18.1\left(\mathrm{CH}_{2} \mathrm{CH}_{2} \mathrm{CH}_{3}, \mathrm{C}-4\right), 13.9\left(\mathrm{CH}_{2} \mathrm{CH}_{2} \mathrm{CH}_{3}, \mathrm{C}-4\right)$; Anal. Calcd for $\mathrm{C}_{20} \mathrm{H}_{26} \mathrm{~N}_{2} \mathrm{O}_{4}$ : C, 67.02; H, 7.31; N, 7.82. Found: C, 67.12; H, 7.37; N, 7.76.

Methyl 2-(4-amino-2-methoxy-6-((phenylamino)methyl)phenoxy)hexanoate (5f) Brown semi-solid This compound (5f) was prepared from methyl 2-(2-methoxy4-nitro-6-((phenylimino)methyl)phenoxy)hexanoate (3f) $(0.40 \mathrm{mmol}, 0.16 \mathrm{~g})$ and $10 \% \mathrm{Pd} / \mathrm{C}$ catalyst $(0.016 \mathrm{~g})$ according to the general procedure. The product obtained as a brown semi-solid $0.11 \mathrm{~g}(74 \%)$; FTIR (neat) $v_{\max }$ : 3370, 3048, 2956, 1740, 1599, 1196, $1055 \mathrm{~cm}^{-1} ;{ }^{1} \mathrm{H}$ NMR $\left(\mathrm{CDCl}_{3}, 400 \mathrm{MHz}\right): \delta=7.27-6.96(\mathrm{~m}, 2 \mathrm{H}, \mathrm{Ar}), 6.87-6.55$ $(\mathrm{m}, 3 \mathrm{H}, \mathrm{Ar}), 6.24(\mathrm{~d}, J=2.5 \mathrm{~Hz}, 1 \mathrm{H}, \mathrm{Ar}), 6.13$ (d, $J=2.5 \mathrm{~Hz}, 1 \mathrm{H}, \mathrm{Ar}), 4.73(\mathrm{t}, J=6.2 \mathrm{~Hz}, 1 \mathrm{H}, \mathrm{CH})$, 
4.40-4.29 (m, 2H, $\mathrm{CH}_{2}$ ), 3.83-3.62 (m, 8H; 6H, $\mathrm{OCH}_{3}, 2 \mathrm{H}$, $\mathrm{NH}_{2}: \mathrm{D}_{2} \mathrm{O}$ exchangeable), 1.98-1.81 (m, 2H, $\left.\mathrm{CH}_{2}\right)$, 1.51-1.21 (m, 5H; 4H, $\mathrm{C}_{2} \mathrm{H}_{4}, 1 \mathrm{H}, \mathrm{NH}: \mathrm{D}_{2} \mathrm{O}$ exchangeable), $0.87\left(\mathrm{t}, \quad J=7.1 \mathrm{~Hz}, 3 \mathrm{H} ; \mathrm{CH}_{3}\right) ;{ }^{13} \mathrm{C} \mathrm{NMR} \quad\left(\mathrm{CDCl}_{3}\right.$, $101 \mathrm{MHz}): \delta=173.0$ (C, C-2), 152.1 (C, C-6), $148.6(\mathrm{C}$, C-13), 142.8 (C, C-8), 137.3 (C, C-5), 133.5 (C, C-10), 129.1 (C, C-15, C-17), 117.1 (C, C-16), 112.9 (C, C-14, C-18), 106.9 (C, C-9), 99.3 (C, C-7), 80.5 (CH, C-3), 55.6 $\left(\mathrm{OCH}_{3}, \mathrm{C}-11\right), 51.7\left(\mathrm{OCH}_{3}, \mathrm{C}-1\right), 43.4\left(\mathrm{CH}_{2}, \mathrm{C}-12\right), 33.1$ $\left(\mathrm{CH}_{2} \mathrm{CH}_{2} \mathrm{CH}_{2} \mathrm{CH}_{3}, \mathrm{C}-4\right), 26.9\left(\mathrm{CH}_{2} \mathrm{CH}_{2} \mathrm{CH}_{2} \mathrm{CH}_{3}, \mathrm{C}-4\right)$, $22.5\left(\mathrm{CH}_{2} \mathrm{CH}_{2} \mathrm{CH}_{2} \mathrm{CH}_{3}, \mathrm{C}-4\right), 13.9\left(\mathrm{CH}_{2} \mathrm{CH}_{2} \mathrm{CH}_{2} \mathrm{CH}_{3}\right.$, C-4); Anal. Calcd for $\mathrm{C}_{21} \mathrm{H}_{28} \mathrm{~N}_{2} \mathrm{O}_{4}$ (372.2): C, 67.72; $\mathrm{H}$, 7.58; N, 7.52. Found: C, 67.68; H, 7.49; N, 7.51.

\section{Microbiological work}

\section{Microorganisms}

The antimicrobial activity of the synthesized compounds was assessed against 11 microbial strains: 5 Gram-negative, Escherichia coli American Type Culture Collection (ATTC) 25922, Escherichia coli (ATCC 8739), Pseudomonas aeruginosa (ATCC 27853), Pseudomonas aeruginosa (wild-type strain isolated from clinical sample), Acinetobacter baumannii (wild-type strain isolated from clinical sample), and 6 Gram-positive, Enterococcus faecalis (ATCC 29212), Staphylococcus aureus MSSA (ATCC 25923), Staphylococcus aureus MRSA (ATCC 43300), Staphylococcus aureus MLSB of inducible phenotype with resistance to macrolide-lincosamide-streptogramin B antibiotics (wild-type strain isolated from clinical sample), Micrococcus luteus Polish Collection of Microorganisms (PCM) 1944, Streptococcus mutans (wildtype strain isolated from dental plaque). Bacterial strains were cultivated in Brain Heart Infusion (BHI, Oxoid) medium at $37^{\circ} \mathrm{C}$ for $24 \mathrm{~h}$. After incubation, microbial suspension was diluted with sterile phosphate-buffered saline (PBS) to $10^{8} \mathrm{CFU} / \mathrm{mL}$ (turbidity = McFarland barium sulfate standard 0.5 ).

\section{Agar diffusion disk method}

The antimicrobial activities of synthesized compounds were determined by disk diffusion method as recommended by National Committee for Clinical Laboratory Standards (NCCLS) (Furtado and Medeiros, 1980). The compounds were evaluated for antimicrobial activity against bacteria on Müeller-Hinton Agar (MHA, Oxoid) medium. The sterile 6-mm filter paper disks (Whatman, no. 2 , Sigma-Aldrich) were impregnated with $10 \mu \mathrm{L}$ of the tested compound at concentration $100 \mathrm{mg} / \mathrm{mL}$ in acetone (POCH) or DMSO (Sigma-Aldrich). The concentration of each tested compound on a disk was $10 \mathrm{mg} / \mathrm{mL}$. The disks were allowed to remain at room temperature until complete diluent evaporation. The disks loaded with tested compounds were placed onto the surface of the proper agar medium (MHA) seeded with the suspension of appropriate test microorganism and incubated for $24 \mathrm{~h}$ at $36^{\circ} \mathrm{C}$. The solvent acetone or DMSO was used as a vehicle control. Commercial antibiotic ciprofloxacin $(5 \mathrm{mg}$ ) (Oxoid) was used as a positive control. The average diameters of the zone of inhibition (in $\mathrm{mm}$ ) were calculated for each tested sample and control. Tests were performed in triplicate.

\section{Minimal inhibitory concentration (MIC) assay}

Compounds that exhibited activity against specific species of bacteria, as determined by the agar diffusion disk method, were further evaluated for their minimal inhibitory concentrations (MIC) using the serial dilution technique. Minimal inhibitory concentrations (MICs) were obtained by measuring the areas of the microbial growth inhibition using the bioautographic assay as described below. Prior to this study, compounds were dissolved in DMSO to make a $300 \mathrm{mg} / \mathrm{mL}$ stock and then diluted to 200, 100, 50, 25, 10, 5 and $1 \mathrm{mg} / \mathrm{mL}$. MIC values were defined as the lowest concentration of each compound that completely inhibited microbial growth. The results were expressed in milligrams per milliliter.

\section{Bioautographic assay}

The bioautographic assay was performed as described by (Valgas et al., 2007). Plates of silica gel (0.2 mm, SigmaAldrich) were seeded in dot blot with $10 \mu \mathrm{L}$ of each compound at concentration $100 \mathrm{mg} / \mathrm{mL}$ in acetone $(\mathrm{POCH})$ or DMSO (Sigma-Aldrich). The concentration of each tested compound in a spot was $10 \mathrm{mg} / \mathrm{mL}$. Sample spots were placed with a micropipette, and the spot diameter was approximately $7 \mathrm{~mm}$. Each sample spot was located approximately $3 \mathrm{~cm}$ apart and away from the edge of TLC plate. As positive control, a solution of $2 \mathrm{mg}$ of chloramphenicol (Fluka) dissolved in $1 \mathrm{~mL}$ of DMSO (SigmaAldrich) was used. As a vehicle control, the solvent acetone or DMSO was used. The controls were applied to plates in the same fashion as tested compounds. The TLC plates were air-dried, covered with MHA $(10 \mathrm{~mL}$ of the medium on $9 \mathrm{~cm}$ diameter Petri plats) containing $100 \mathrm{~mL}$ of test microorganism suspensions, and incubated for $24 \mathrm{~h}$ at $36{ }^{\circ} \mathrm{C}$. After incubation, in order to visualize zones of growth inhibition, $2 \mathrm{~mL}$ of MTT solution (1 mg of 3-(4,5dimethylthiazol-2-yl)-2,5-diphenyltetrazolium bromide in one $\mathrm{mL}$ sterile physiological solution, Sigma-Aldrich) was 
added to each Petri plate and incubation was continued for $3 \mathrm{~h}$ at the same culture condition. In the last step, the areas of growth inhibition were measured for each tested compound and controls and the average diameters of the zone of inhibition (in $\mathrm{mm}$ ) were calculated. Tests were performed in triplicate.

Acknowledgments The authors would like to thank Prof. Mirosława El Fray, Division of Biomaterials and Microbiological Technologies of West Pomeranian University of Technology, Szczecin, for access to Fourier transform infrared spectroscopy and Peter Sobolewski, Ph.D. for proof-reading and editing the manuscript.

Open Access This article is distributed under the terms of the Creative Commons Attribution 4.0 International License (http:// creativecommons.org/licenses/by/4.0/), which permits unrestricted use, distribution, and reproduction in any medium, provided you give appropriate credit to the original author(s) and the source, provide a link to the Creative Commons license, and indicate if changes were made.

\section{References}

Abdel-Magid AF, Mehrman SJ (2006) A review on the use of sodium triacetoxyborohydride in the reductive amination of ketones and aldehydes. Org Proc Res Dev 10:971-1031

Abdel-Magid AF, Carson KG, Harris BD, Maryanoff CA, Shah RD (1996) Reductive amination of aldehydes and ketones with sodium triacetoxyborohydride. Studies on direct and indirect reductive amination procedures. J Org Chem 61:3849-3862

Alinezhad H, Tajbakhsh M, Hamidi N (2010) Direct reductive amination of carbonyl compounds using sodium borohydridesilica chloride. Turk J Chem 34:307-312

Bala V, Chhonker YS, Hashim SR (2010) Synthesis and antimicrobial activity of Schiff bases derived from 2-formylphenoxy acetic acid. Asian J Chem 22:3447-3452

Beveridge TJ (1999) Structures of gram-negative cell walls and their derived membrane vesicles. J Bacteriol 181:4725-4733

Borch RF, Bernstein MD, Durst HD (1971) The cyanohydridoborate anion as a selective reducing agent. $J$ Am Chem Soc 93:2897-2904

Cheng L-X, Tang J-J, Luo H, Jin X-J, Dai F, Yang Y, Qian Y-P (2010) Antioxidant and antiproliferative activities of hydroxylsubstituted Schiff bases. Bioorg Med Chem Lett 20:2417-2420

Cho BT, Kang SK (2005) Direct and indirect reductive amination of aldehydes and ketones with solid acid-activated sodium borohydride under solvent-free conditions. Tetrahedron 61:5725-5734

Curtis B, Payne TJ, Ash DE, Mohanty DK (2013) Secondary amines containing one aromatic nitro group: preparation, nitrosation, sustained nitric oxide release, and the synergistic effects of released nitric oxide and an arginase inhibitor on vascular smooth muscle cell proliferation. Bioorg Med Chem 21:1123-1135

da Silva CM, da Silva DL, Modolo LV, Alves RB, de Resende MA, Martins CVB, de Fátima Â (2011) Schiff bases: a short review of their antimicrobial activities. J Adv Res 2:1-8

Furtado GL, Medeiros AA (1980) Single-disk diffusion testing (KirbyBauer) of susceptibility of Proteus mirabilis to chloramphenicol: significance of the intermediate category. J Clin Microbiol 12:550-553
Gomez S, Peters JA, Maschmeyer T (2002) The reductive amination of aldehydes and ketones and the hydrogenation of nitriles: mechanistic aspects and selectivity control. Adv Synth Catal 344:1037-1057

Grenga PN, Sumbler BL, Beland F, Priefer R (2009) Reductive amination agents: comparison of $\mathrm{Na}(\mathrm{CN}) \mathrm{BH}_{3}$ and $\mathrm{Si}-\mathrm{CBH}$. Tetrahedron Lett 50:6658-6660

Gribble GW (2006) The synthetic versatility of acyloxyborohydrides. Org Proc Res Dev 10:1062-1075

Hullar TL, Failla DL (1969) Pyridoxal phosphate. II. Benzene analogs. 2-Formylphenoxyacetic acids as potential antimetabolites of pyridoxal phosphate. J Med Chem 12:420-424

Iqbal A, Siddiqui HL, Ashraf CM, Ahmad M, Weaver GW (2007) Synthesis, characterization and antibacterial activity of azomethine derivatives derived from 2-formylphenoxyacetic acid. Molecules 12:245-254

Kitahara T, Koyama N, Matsuda J, Aoyama Y, Hirakata Y, Kamihira S, Kohno S, Nakashima M, Sasaki H (2004) Antimicrobial activity of saturated fatty acids and fatty amines against methicillin-resistant Staphylococcus aureus. Biol Pharm Bull 27:1321-1326

Klyuev MV, Khidekel ML (1980) Catalytic amination of alcohols, aldehydes, and ketones. Russ Chem Rev 49:14-27

Kumar GSS, Kumaresan S (2012) Potash alum [KAl $\left.\left(\mathrm{SO}_{4}\right)_{2} \cdot 12 \mathrm{H}_{2} \mathrm{O}\right]$ catalysed esterification of formylphenoxyaliphatic acids. J Chem Sci 124:857-863

Kwiecień H (2004) Synthesis of 2-alkyl-5-nitrobenzofurans via 2-(2formyl-4-nitrophenoxy)alkanoic acids. Pol J Chem 78:18651869

Kwiecień H, Szychowska M (2007) Simple synthesis of 7-amino-4,5dihydrobenzo[f][1,4] oxazepin-3-ones. Synth Commun 37:3599-3609

Mohini Y, Prasad RBN, Karuna MSL (2013) Synthesis of fatty acid Schiff base esters as potential antimicrobial and chemotherapeutic agents. Med Chem Res 22:4360-4366

Neidigh KA, Avery MA, Williamson JS, Bhattacharyya S (1998) Facile preparation of $N$-methyl secondary amines by titanium(IV) isopropoxide-mediated reductive amination of carbonyl compounds. J Chem Soc Perkin Trans 1:2527-2532

Panfilov AV, Markovich YuD, Ivashev IP, Zhirov AA, Eleev AF, Kurochkin VK, Kirsanov AT, Nazarov GV (2000) Sodium borohydride in reductive amination reactions. Pharm Chem $\mathrm{J}$ 34:76-78

Pattan SR, Hullolikar RL, Pattan JS, Kapadnis BP, Dighe NS, Dengale SS, Nikalje A, Nirmal SA (2009) Synthesis and evaluation of some new pyrazolo phenoxy acetic acid derivatves for their antitubercular activity. J Pharm Sci Res 3:63-68

Petrisko M, Krupka J (2005) Isomerization of an imine intermediate in a reductive amination reaction over metal catalysts. Res Chem Intermed 31:769-778

Saidi MR, Brown RS, Ziyaei-Halimjani A (2007) Reductive amination of aldehydes with sodium borohydride and lithium aluminum hydride in the presence of lithium perchlorate. J Iran Chem Soc 4:194-198

Saxena I, Borah R, Sarma JC (2000) Reductive amination of aromatic aldehydes and ketones with nickel boride. J Chem Soc Perkin Trans 1:503-504

Shi L, Ge H-M, Tan S-H, Li H-Q, Song Y-C, Zhu H-L, Tan R-X (2007) Synthesis and antimicrobial activities of Schiff bases derived from 5-chloro-salicylaldehyde. Eur J Med Chem 42:558-564

Singh P, Negi JS, Rawat MSM, Pant GJ, Bishoyi AK (2011) Syntheses, characterization and antimicrobial activity of 3-(aminophenyl)-1,3-diphenylpropanones, novel aza-michael products. Int J Chem Tech Res 3:584-589 
Sztanke K, Maziarka A, Osinka A, Sztanke M (2013) An insight into synthetic Schiff bases revealing antiproliferative activities in vitro. Bioorg Med Chem 21:3648-3666

Tarasevich VA, Kozlov NG (1999) Reductive amination of oxygencontaining organic compounds. Russ Chem Rev 68:55-72

Tripathi RP, Verma SS, Pandey J, Tiwari VK (2008) Recent development on catalytic reductive amination and applications. Curr Org Chem 12:1093-1115
Valgas C, de Souza SM, Smania EFA, Smania JA (2007) Screening methods to determine antibacterial activity of natural products. Braz. J Microbiol 38:369-380 